\title{
A supersymmetric electroweak scale seesaw model
}

\author{
Jung Chang, ${ }^{a, b}$ Kingman Cheung, ${ }^{b, c, d}$ Hiroyuki Ishida, ${ }^{b}$ Chih-Ting Lu, ${ }^{c}$ \\ Martin Spinrath ${ }^{b}$ and Yue-Lin Sming Tsai ${ }^{b}$ \\ ${ }^{a}$ Department of Physics, Chonnam National University, \\ 300 Yongbong-dong, \\ Buk-gu, Gwangju, 500-757, Republic of Korea \\ ${ }^{b}$ Physics Division, National Center for Theoretical Sciences, \\ Hsinchu 30013, Taiwan \\ ${ }^{c}$ Department of Physics, National Tsing Hua University, \\ Hsinchu 30013, Taiwan \\ ${ }^{d}$ Division of Quantum Phases 8 Devices, School of Physics, Konkuk University, \\ Seoul 143-701, Republic of Korea \\ E-mail: lovejesus99wwjd@gmail.com, cheung@phys.nthu.edu.tw, \\ hiroyuki403@cts.nthu.edu.tw, timluyu@hotmail.com, \\ martin.spinrath@cts.nthu.edu.tw, sming.tsai@cts.nthu.edu.tw
}

ABSTRACT: In this paper we propose a novel supersymmetric inverse seesaw model which has only one additional $Z_{6}$ symmetry. The field content is minimal to get a viable neutrino spectrum at tree-level. Interestingly, the inverse seesaw scale in our model is related to the scale of electroweak symmetry breaking. Due to that origin we are less biased about hierarchies and discuss three different types of the inverse seesaw mechanism with different phenomenologies. We can successfully reproduce neutrino masses and mixing and our model is consistent with current bounds on neutrinoless double beta decay, non-unitarity of the PMNS matrix and charged lepton flavor violation.

KeYwords: Beyond Standard Model, Neutrino Physics, Supersymmetric Standard Model

ARXIV EPRINT: 1707.04374 


\section{Contents}

1 Introduction $\quad 1$

2 The model $\quad 4$

2.1 The superpotential 4

2.2 The soft SUSY breaking terms and the scalar potential 5

3 Phenomenology $\quad 7$

$\begin{array}{lll}3.1 & \text { Leptonic masses and mixing } & 7\end{array}$

$\begin{array}{llr}3.1 .1 & \text { ISS type I } & 9\end{array}$

$\begin{array}{lll}3.1 .2 & \text { ISS type II } & 10\end{array}$

3.1.3 ISS type III 11

$\begin{array}{lll}3.1 .4 & \text { Non-unitarity of the mixing matrix } & 12\end{array}$

3.1.5 The Yukawa couplings 12

$\begin{array}{ll}3.2 & \text { Neutrinoless double beta decay } \\ & \text { Charged }\end{array}$

$\begin{array}{lll}3.3 & \text { Charged lepton flavor violation } & 14\end{array}$

$\begin{array}{lll}\text { 3.3.1 The non-SUSY part } & 15\end{array}$

$\begin{array}{lll}3.3 .2 & \text { The SUSY part } & 16\end{array}$

$\begin{array}{lll}4 & \text { Summary and conclusions } & 19\end{array}$

A Explicit expressions for mixing of light and heavy neutrinos 21

\section{Introduction}

Supersymmetry (SUSY) is still one of the most attractive models for physics beyond the Standard Model (SM). It not only solves the gauge hierarchy problem, but also provides a dynamical mechanism for electroweak symmetry breaking. The minimal supersymmetric extension of the SM, called the minimal supersymmetric standard model (MSSM) [1], has roughly doubled the degrees of freedom of the SM and has many phenomenological implications for, e.g. Higgs and flavor physics. The MSSM is usually defined with an adhoc $Z_{2}$ symmetry, known as $R$-parity, which can provide a dark matter (DM) candidate to explain the DM relic density of the universe.

Another undeniable evidence of physics beyond the SM is neutrino masses and oscillations [2]. Although the MSSM with explicit $R$-parity violation could explain neutrino masses [3], the virtue of having a DM candidate would then be lost in general. Therefore, if one insists on having a DM candidate in the MSSM, one has to include additional fields or particles, e.g. right-handed neutrinos, in order to generate neutrino masses and oscillations. 
One of the most celebrated ways to generate neutrino masses is the seesaw mechanism [4-11], which is often considered to be the most natural and attractive. The benefit and drawback of the original seesaw is that its scale is generically around the scale of grand unified theories (GUTs) $\sim 10^{12-16} \mathrm{GeV}$, which is not accessible for direct phenomenological tests. There have been many alternatives or modifications to the original seesaw such that the seesaw scale can be as low as $\mathrm{GeV}$ or $\mathrm{TeV}$ which can be tested in current or future experiments. Nevertheless, one big drawback of many of these models is that the seesaw scale is put in rather ad-hoc by hand to be low.

One popular variant of these proposals is the inverse seesaw (ISS) mechanism [12, 13], which is an extension of the original seesaw model but with a much lower mass scale usually below several $\mathrm{TeV}$. The inverse seesaw mechanism generates small neutrino masses with rather large Yukawa couplings and violates lepton number mildly. At such a low scale the model can be tested at Hadron colliders (the LHC and at future $100 \mathrm{TeV}$ colliders, e.g. [14-16]) and future high energy lepton colliders (the Circular Electron Positron Collider, e.g. [17, 18], International Linear Collider, e.g. [17, 18], and the FCC-ee, e.g. [19]), for an overview and comparison of the different collider possibilities, see, e.g. [20]. Indirect effects can also be tested at low-energy flavor physics experiments, e.g. [21], or in Higgs decays, e.g. [22].

There is a plethora of ISS models and not surprisingly we are by far not the first to discuss a supersymmetric version. For the sake of brevity we give here a short overview of SUSY ISS models only. To our knowledge these models can be roughly categorized under one of the four categories where each time the additional fields required in the ISS have to be added: (i) MSSM [23-26], (ii) MSSM/NMSSM with extended gauge symmetry [27-32], (iii) NMSSM [17,33], and (iv) supersymmetric Left-Right symmetry model [34, 35]. We briefly summarize these models as follows.

MSSM with additional gauge singlets: it has been pointed out in ref. [26] that by adding only one pair of gauge singlets $(S, N)$ to the MSSM it is sufficient to explain the neutrino data using an ISS. One neutrino mass is generated at tree level while the other non-zero neutrino masses are generated by loop effects. This is justifiable called the minimal version of SUSY inverse seesaw model. In our approach we also aim for minimality but we want to explain all neutrino masses at tree-level already which forces us to introduce two pairs of extra gauge singlets. Of course it is also possible and popular to introduce three pairs of the extra gauge singlets with opposite lepton numbers, see, e.g. [23-25].

The gauge extended SUSY: in this class of models, the seesaw mechanism is derived from a symmetry breaking pattern of a $B-L$ extension of the MSSM. The gauge group is $\mathrm{SU}(3)_{C} \times \mathrm{SU}(2)_{L} \times \mathrm{U}(1)_{Y} \times \mathrm{U}(1)_{B-L}$, which gives rise to three SM singlets due to the $\mathrm{U}(1)_{B-L}$ anomaly cancellation conditions. These singlets can be the right-hand neutrinos for the seesaw mechanism. At the same time, the lightest right-handed sneutrino could be the lightest SUSY particle (LSP) [27]. This is also attractive since it can be embedded into $\mathrm{SO}(10)$ which was studied, e.g. in refs. [28-30], where even an additional gauge factor $\mathrm{U}(1)_{R}$ is introduced. Interestingly, the sneutrino in 
this class of models can survive all the dark matter constraints in the inverse seesaw extension but not in the linear seesaw.

The NMSSM with an extra singlet sector: by adding an extra singlet sector to the NMSSM [33], tiny neutrino masses can be radiatively generated by the SUSY breaking parameters at very low scales similar to [26] which we mentioned above. In such a model, the sneutrino or the lightest neutralino could be the LSP $[17,33]$. In another NMSSM extension $[31,32]$ they connect neutrino physics to asymmetric dark matter.

$\mathrm{SU}(2)_{L} \times \mathrm{SU}(2)_{R} \times \mathrm{U}(1)_{B-L}$ : in the last class of supersymmetric inverse seesaw implementations the MSSM gauge group is extended to a left-right symmetry $\mathrm{SU}(2)_{L} \times$ $\mathrm{SU}(2)_{R} \times \mathrm{U}(1)_{B-L}[34,35]$. In these models the $B-L$ symmetry is broken at a low scale $\sim \mathrm{TeV}$ and the neutrino masses are dynamically generated. Interestingly these models can be embedded into $\mathrm{SO}(10)$ models which reduces the effective number of parameters making the model more predictive.

In this paper we discuss a supersymmetric version of the ISS where a $Z_{6}$ symmetry plays the role of lepton number which is usually implemented as an approximate symmetry in ISS models. Conventionally, $R$-parity is introduced to ensure proton stability. This is not needed in our model since all $R$-parity violating operators are already forbidden by the $Z_{6}$-symmetry. The $Z_{6}$ is broken in the same way as the electroweak symmetry in the MSSM and both scales are related to SUSY breaking. Hence, in our model we have an intimate connection between the seesaw scale and the $\mathrm{TeV}$ scale which gives a strong theoretical motivation to have a low seesaw scale.

Our model is minimal not only with respect to symmetry extensions but also with respect to the field content. Only five additional SM singlet fields are introduced to the superpotential. The superfields $\hat{N}^{c}$ contain right-handed (RH) neutrinos and sneutrinos while the singlet superfields $\hat{S}$ and $\hat{X}$ contain new singlet scalars and fermions. Unlike the NMSSM, in which the new singlet superfield also couples to the two Higgs-doublet superfields, here the singlet superfields $\hat{S}$ and $\hat{X}$ only couple to the RH neutrino superfields $\hat{N}^{c}$ or to themselves. Our model hence would fall most closely under the first category of an ISS extension of the MSSM since our additional symmetry is not gauged.

Since we gave up on some rather ad-hoc arguments about the scales involved in the ISS we do not have to restrict ourselves to the original inverse seesaw mechanism with $M_{S} \ll M_{D} \ll \mu_{\mathrm{NS}}$, where $M_{S}$ is the singlets mass term, $M_{D}$ the Dirac neutrino mass term and $\mu_{\mathrm{NS}}$ a supersymmetric mass term respectively. To remind the reader the ISS neutrino mass matrix has the structure

$$
M_{\nu}=\left(\begin{array}{ccc}
0 & M_{D} & 0 \\
M_{D}^{T} & 0 & \mu_{\mathrm{NS}} \\
0 & \mu_{\mathrm{NS}}^{T} & M_{S}
\end{array}\right)
$$

in the basis $\left(\nu, N^{c}, S\right)^{T}$. In our setup the original ordering of mass hierarchies can be easily generalized to three different types of inverse seesaw mechanisms: (i) $M_{S} \ll M_{D} \ll \mu_{\mathrm{NS}}$ 


\begin{tabular}{|ccccccccccc|}
\hline Superfield & $\hat{Q}_{i}$ & $\hat{U}_{i}^{c}$ & $\hat{E}_{i}^{c}$ & $\hat{L}_{i}$ & $\hat{D}_{i}^{c}$ & $\hat{H}_{u}$ & $\hat{H}_{d}$ & $\hat{N}_{\alpha}^{c}$ & $\hat{S}_{\alpha}$ & $\hat{X}$ \\
\hline$Z_{6}$ charge & 5 & 5 & 5 & 3 & 3 & 2 & 4 & 1 & 5 & 2 \\
\hline
\end{tabular}

Table 1. Superfield content of the model and charge assignment under the additional discrete $Z_{6}$ symmetry. The new superfields compared to the MSSM, $\hat{N}^{c}, \hat{S}$ and $\hat{X}$, are singlets under the Standard Model gauge group. The indices $i=1,2,3$ and $\alpha=1,2$ are generation indices.

(ISS type I), (ii) $M_{S} \approx M_{D} \ll \mu_{\mathrm{NS}}$ (ISS type II), and (iii) $M_{D} \ll M_{S} \ll \mu_{\mathrm{NS}}$ (ISS type III). We investigate these three types of the ISS and find that they can have very different phenomenology which is expected since, for instance, the Yukawa couplings turn out to be very different in size.

This work is organized as follows: first we describe the model in section 2 and in section 3 we discuss various phenomenological implications such as the neutrino mass spectrum and mixing, neutrinoless double beta decay, and charged lepton-flavor violations. We summarize and conclude in section 4 . In the appendix we have collected some explicit expressions for mixing matrices which are too long for the main text.

\section{The model}

In this section, we describe the model in detail, that is the superpotential, the soft SUSY breaking parameters, and the scalar potential. The aim is to construct a minimal supersymmetric inverse seesaw model. It is minimal in the sense that we want to extend the MSSM with the least possible extra fields and symmetries to get a viable inverse seesaw mechanism to generate neutrino masses at tree level which will be discussed in the next section.

\subsection{The superpotential}

We impose a $Z_{6}$ symmetry on the superpotential under which the superfields transform as

$$
\hat{\Phi} \rightarrow \hat{\Phi} \exp \left[\mathrm{i} q \frac{2 \pi}{6}\right]
$$

where $q$ runs from 0 to 5 . The assignment of $q$ for the superfields in our model is listed in table 1.

This charge assignment is not unique but we have chosen the $Z_{6}$ charges such that they are compatible with $\mathrm{SU}(5)$ unification and such that we forbid the $R$-parity violating operators of the MSSM. Because our superpotential does not conserve U(1) lepton number, $R$-parity is not well defined.

The renormalizable superpotential compatible with the SM gauge symmetries and the $Z_{6}$ symmetry is then given by

$$
\mathcal{W}=\mathcal{W}_{\mathrm{MSSM}}+\mathcal{W}_{\nu}
$$

where

$$
\begin{aligned}
\mathcal{W}_{\mathrm{MSSM}} & =Y_{u} \hat{Q} \hat{H}_{u} \hat{U}^{c}-Y_{d} \hat{Q} \hat{H}_{d} \hat{D}^{c}-Y_{e} \hat{L} \hat{H}_{d} \hat{E}^{c}+\mu_{H} \hat{H}_{u} \hat{H}_{d} \\
\mathcal{W}_{\nu} & =Y_{\nu} \hat{L} \hat{H}_{u} \hat{N}^{c}+\mu_{\mathrm{NS}} \hat{N}^{c} \hat{S}+\frac{\lambda}{2} \hat{X} \hat{S}^{2}+\frac{\kappa}{3} \hat{X}^{3}
\end{aligned}
$$


and we have suppressed generation indices. In our conventions, we label the superfields with a hat, the fermionic components of the matter fields (including $\hat{N}^{c}$ and $\hat{X}$ ) without hat and their scalar components with a tilde. This is twisted for the Higgs doublets and $\hat{X}$ (scalars without hat or tilde and fermions with a tilde). Note that the superfields $\hat{N}^{c}$ will give rise to right-handed neutrinos and sneutrinos while the singlet superfields $\hat{S}$ and $\hat{X}$ will give rise to new singlet scalars and fermions.

For the MSSM fields we assume the conventional number of generations. To accommodate the neutrino masses and mixing at tree level we need at least two generations of right-handed neutrino superfields $\hat{N}^{c}$ and two generations of additional singlet superfields $\hat{S}$ for the realization of the ISS mechanism, see also [36]. For the sake of simplicity this is what we assume throughout the rest of the paper. $\hat{X}$ gives rise to lepton number violation and its vacuum expectation value (vev) induces a Majorana mass term of $S$ as we will see in the next section.

\subsection{The soft SUSY breaking terms and the scalar potential}

The soft SUSY breaking terms can be grouped into the ordinary MSSM part and additional terms

$$
-\mathcal{L}_{\text {soft }}=-\mathcal{L}_{\text {soft }, \text { MSSM }}-\mathcal{L}_{\text {soft }, \nu}
$$

where

$$
\begin{aligned}
-\mathcal{L}_{\text {soft }, \mathrm{MSSM}}= & \frac{1}{2} M_{1} \tilde{B} \tilde{B}+\frac{1}{2} M_{2} \tilde{W} \tilde{W}+\frac{1}{2} M_{3} \tilde{g} \tilde{g} \\
& +M_{\tilde{Q}}^{2} \tilde{Q}^{\dagger} \tilde{Q}+M_{\tilde{U}^{c}}^{2} \tilde{U}^{\dagger} \tilde{U}_{c}+M_{\tilde{D}^{c}}^{2} \tilde{D}^{c^{\dagger}} \tilde{D}_{c}+M_{\tilde{L}}^{2} \tilde{L}^{\dagger} \tilde{L}+M_{\tilde{E}^{c}}^{2} \tilde{E}^{c^{\dagger}} \tilde{E}^{c} \\
& +M_{H_{u}}^{2} H_{u}^{\dagger} H_{u}+M_{H_{d}}^{2} H_{d}^{\dagger} H_{d}+\left(b_{H} H_{u} H_{d}+\text { H.c. }\right) \\
& +\left(A_{u} \tilde{Q} H_{u} \tilde{U}^{c}-A_{d} \tilde{Q} H_{d} \tilde{D}^{c}-A_{e} \tilde{L} H_{d} \tilde{E}^{c}+\text { H.c. }\right) \\
-\mathcal{L}_{\text {soft }, \nu}= & M_{\tilde{N}^{c}}^{2} \tilde{N}^{c \dagger} \tilde{N}^{c}+M_{\tilde{S}}^{2} \tilde{S}^{\dagger} \tilde{S}+M_{X}^{2} X^{\dagger} X+\left(b_{\mathrm{NS}} \tilde{N}^{c} \tilde{S}+\text { H.c. }\right) \\
& +\left(A_{\nu} \tilde{L} H_{u} \tilde{N}^{c}+\frac{1}{2} A_{\lambda} X \tilde{S}^{2}+\frac{1}{3} A_{\kappa} X^{3}+\text { H.c. }\right)
\end{aligned}
$$

and where we have suppressed any gauge or generation indices.

To discuss the scalar potential we still have to add the $D$ - and $F$-terms. Since the new states do not have gauge interactions we only have to consider the $F$-terms for them which are given by $\sum_{i}\left|\partial W / \partial \phi_{i}\right|^{2}$, where $\phi_{i}$ is the scalar component of the superfields to be considered. The new part of the scalar potential then reads

$$
\begin{aligned}
V_{\text {new }}= & \left|Y_{\nu} \tilde{L} H_{u}+\mu_{\mathrm{NS}} \tilde{S}\right|^{2}+\left|\mu_{\mathrm{NS}} \tilde{N}^{c}+\lambda X \tilde{S}\right|^{2}+\left|-Y_{e} H_{d} \tilde{E}^{c}+Y_{\nu} H_{u} \tilde{N}^{c}\right|^{2} \\
& +\left|\frac{1}{2} \lambda \tilde{S}^{2}+\kappa X^{2}\right|^{2}+M_{\tilde{N}^{c}}^{2} \tilde{N}^{\dagger} \tilde{N}^{c}+M_{\tilde{S}}^{2} \tilde{S}^{\dagger} \tilde{S}+M_{X}^{2} X^{\dagger} X+\left(b_{\mathrm{NS}} \tilde{N}^{c} \tilde{S}+\text { H.c. }\right) \\
& +\left(A_{\nu} \tilde{L} H_{u} \tilde{N}^{c}+\frac{1}{2} A_{\lambda} X \tilde{S}^{2}+\frac{1}{3} A_{\kappa} X^{3}+\text { H.c. }\right)
\end{aligned}
$$

This is in general a very complicated potential since we have to consider three generations of $\tilde{L}$ and $\tilde{E}^{c}$, two generations of $\tilde{N}^{c}$, two generations of $\tilde{S}$, and one generation of $X$. In 
addition, this potential mixes with the conventional MSSM potential. Before we study this in more detail we will restrict ourselves to the case of one generation of slepton doublets, right-handed sneutrinos and scalar singlets each. We also assume that all couplings and mass parameters are real which allows us to understand some essential features and the rest is left to a future detailed numerical study of the model.

Since we do not want to introduce any additional source of electroweak symmetry breaking we set $\langle\tilde{L}\rangle=0$ and $\left\langle\tilde{E}^{c}\right\rangle=0$. Keep in mind that choosing the appropriate parameters this is always possible, since there is a $D$-term quartic in $\tilde{L}$ and a $D$-term quartic in $\tilde{E}^{c}$ which dominates the potential for large field values and the other parameters can be adjusted to allow only the trivial vacuum.

We define the vev of the relevant scalar fields as $\left\langle H_{u}^{0}\right\rangle=v_{u},\left\langle H_{d}^{0}\right\rangle=v_{d},\left\langle\tilde{N}^{c}\right\rangle=v_{N}$, $\langle\tilde{S}\rangle=v_{S}$, and $\langle X\rangle=v_{X}$. The scalar potential is

$$
\begin{aligned}
V_{\mathrm{scalar}} \supset & \left(M_{H_{u}}^{2}+\mu_{H}^{2}\right) v_{u}^{2}+\left(M_{H_{d}}^{2}+\mu_{H}^{2}\right) v_{d}^{2}-2 b_{H} v_{u} v_{d}+\frac{1}{8}\left(g^{2}+g^{\prime 2}\right)\left(v_{u}^{2}-v_{d}^{2}\right)^{2} \\
& +\left(\mu_{\mathrm{NS}} v_{S}\right)^{2}+\left(\mu_{\mathrm{NS}} v_{N}+\lambda v_{S} v_{X}\right)^{2}+\left(Y_{\nu} v_{u} v_{N}\right)^{2}+\left(\frac{1}{2} \lambda v_{S}^{2}+\kappa v_{X}^{2}\right)^{2} \\
& +M_{\tilde{N}^{c}}^{2} v_{N}^{2}+M_{\tilde{S}}^{2} v_{S}^{2}+M_{X}^{2} v_{X}^{2}+2\left(b_{\mathrm{NS}} v_{N} v_{S}+\frac{1}{2} A_{\lambda} v_{X} v_{S}^{2}+\frac{1}{3} A_{\kappa} v_{X}^{3}\right) \\
& =m_{H_{u}}^{2} v_{u}^{2}+Y_{\nu}^{2} v_{N}^{2} v_{u}^{2}+m_{H_{d}}^{2} v_{d}^{2}-2 b_{H} v_{u} v_{d}+\frac{1}{8}\left(g^{2}+g^{\prime 2}\right)\left(v_{u}^{2}-v_{d}^{2}\right)^{2} \\
& +m_{S}^{2} v_{S}^{2}+m_{N}^{2} v_{N}^{2}+M_{X}^{2} v_{X}^{2}+v_{N} v_{S}\left(2 b_{\mathrm{NS}}+2 \lambda \mu_{\mathrm{NS}} v_{X}\right)+A_{\lambda} v_{X} v_{S}^{2} \\
& +\frac{2}{3} A_{\kappa} v_{X}^{3}+\frac{1}{4} \lambda^{2} v_{S}^{4}+\kappa^{2} v_{X}^{4}+\left(\lambda^{2}+\lambda \kappa\right) v_{S}^{2} v_{X}^{2}
\end{aligned}
$$

where we have set $m_{H_{u}}^{2}=M_{H_{u}}^{2}+\mu_{H}^{2}, m_{H_{d}}^{2}=M_{H_{d}}^{2}+\mu_{H}^{2}, m_{S}^{2}=M_{\tilde{S}}^{2}+\mu_{\mathrm{NS}}^{2}$ and $m_{N}^{2}=$ $M_{\tilde{N}^{c}}^{2}+\mu_{\mathrm{NS}}^{2}$. The conventional MSSM Higgs part was taken from [37].

Now we are looking at the first derivatives to look for extrema of the potential

$$
\begin{aligned}
& \frac{\partial V_{\text {scalar }}}{\partial v_{u}}=2\left(m_{H_{u}}^{2}+Y_{\nu}^{2} v_{N}^{2}\right) v_{u}-2 b_{H} v_{d}+\frac{1}{2}\left(g^{2}+g^{\prime 2}\right)\left(v_{u}^{3}-v_{u} v_{d}^{2}\right)=0 \\
& \frac{\partial V_{\text {scalar }}}{\partial v_{d}}=2 m_{H_{d}}^{2} v_{d}-2 b_{H} v_{u}+\frac{1}{2}\left(g^{2}+g^{\prime 2}\right)\left(v_{d}^{3}-v_{u}^{2} v_{d}\right)=0 \\
& \frac{\partial V_{\text {scalar }}}{\partial v_{S}}=2 m_{S}^{2} v_{S}+v_{N}\left(2 b_{\mathrm{NS}}+2 \lambda \mu_{\mathrm{NS}} v_{X}\right)+2 A_{\lambda} v_{X} v_{S}+\lambda^{2} v_{S}^{3}+2\left(\lambda^{2}+\lambda \kappa\right) v_{S} v_{X}^{2}=0 \\
& \frac{\partial V_{\text {scalar }}}{\partial v_{N}}=2\left(m_{N}^{2}+Y_{\nu}^{2} v_{u}^{2}\right) v_{N}+\left(2 b_{\mathrm{NS}}+2 \lambda \mu_{\mathrm{NS}} v_{X}\right) v_{S}=0 \\
& \frac{\partial V_{\mathrm{scalar}}}{\partial v_{X}}=2 M_{X}^{2} v_{X}+2 \lambda \mu_{\mathrm{NS}} v_{N} v_{S}+A_{\lambda} v_{S}^{2}+2 A_{\kappa} v_{X}^{2}+4 \kappa^{2} v_{X}^{3}+2\left(\lambda^{2}+\lambda \kappa\right) v_{S}^{2} v_{X}=0
\end{aligned}
$$

Here, we would like to note several features of these tadpole conditions in eqs. (2.10)-(2.14). Once we switch off the vevs of the additional fields, i.e. $v_{N}=v_{S}=v_{X}=0$, these tadpole conditions go back to the MSSM ones. 
The only viable solution from a phenomenological point of view of these tadpole conditions is $v_{N}=v_{S}=0$ and $v_{X} \neq 0$. In particular we need $v_{X} \neq 0$ to generate neutrino masses. Its solution is

$$
v_{X}=-\frac{A_{\kappa}}{4 \kappa^{2}} \pm \frac{\sqrt{A_{\kappa}^{2}-8 \kappa^{2} M_{X}^{2}}}{4 \kappa^{2}} .
$$

This will be important later on and tells us that in our setup the neutrino mass scale is related to the scale of SUSY breaking which is different from many ISS models where the right-handed neutrino masses are forbidden and the smallness of the fermionic singlet masses are put in by hand due to the approximate lepton number conservation. Therefore, our setup is minimal and we can derive all the masses without any willful assumption.

In principle one can now also discuss the second derivatives and study the conditions for the potential to have a minimum but we do not find any simple, important insights from there. In particular, the case above is a simplified version of the model under study and the expressions get very lengthy for a more realistic case. For the later discussion we just keep in mind that $X$ gets an electroweak scale (= SUSY breaking scale) vev but $\tilde{S}$ and $\tilde{N}^{c}$ do not receive a vev.

\section{Phenomenology}

In this section, we discuss some phenomenological aspects of our model. Like in any supersymmetric model there is a huge amount of phenomenological aspects which could be discussed. In this work, we focus only on the features immediately related to neutrino masses and mixing. That is the non-unitarity of the Pontecorvo-Maki-Nakagawa-Sakata (PMNS) matrix, neutrinoless double beta decay and charged-lepton flavor violations (cLFV). Other aspects will be discussed in future publications.

\subsection{Leptonic masses and mixing}

We begin with the relevant Yukawa couplings and mass terms relevant to the leptonic sector in the Lagrangian

$$
-\mathcal{L}_{\nu}=-\left(Y_{e}\right)_{i j} L_{i} H_{d} E_{j}^{c}+\left(Y_{\nu}\right)_{i \alpha} L_{i} N_{\alpha}^{c} H_{u}+\left(\mu_{\mathrm{NS}}\right)_{\alpha \beta} N_{\alpha}^{c} S_{\beta}+\frac{1}{2} \lambda_{\alpha \beta} S_{\alpha} S_{\beta} X+\text { H.c. },
$$

where $i, j=1,2,3$ and $\alpha, \beta=1,2$. We are working in a basis where the charged-lepton Yukawa couplings are diagonal and

$$
m_{l}=y_{l} v \cos \beta
$$

where $l=e, \mu, \tau, v=174 \mathrm{GeV}$ and $v \cos \beta=\left\langle H_{d}^{0}\right\rangle$.

Since $X$ receives a vev we define the mass matrix

$$
M_{S}=\lambda v_{X}
$$

Note that $M_{S}$ is symmetric since it is a Majorana mass matrix. 
We also define a Dirac neutrino mass matrix for the neutrinos

$$
M_{D}=Y_{\nu} v \sin \beta,
$$

where $v \sin \beta=\left\langle H_{u}^{0}\right\rangle$. Furthermore, the mixing term between $N^{c}$ and $S$ is $\mu_{\mathrm{NS}}$. Using these definitions it is easy to write down the full neutrino mass matrix

$$
M_{\nu}=\left(\begin{array}{ccc}
0 & M_{D} & 0 \\
M_{D}^{T} & 0 & \mu_{\mathrm{NS}} \\
0 & \mu_{\mathrm{NS}}^{T} & M_{S}
\end{array}\right)
$$

in the basis $\left(\nu, N^{c}, S\right)^{T}$. One will immediately recognise that this is the pattern of a double or an inverse seesaw mechanism [13]. The double seesaw mechanism requires $M_{S} \gg \mu_{\mathrm{NS}}$ whereas the inverse seesaw mechanism requires $M_{S} \ll \mu_{\mathrm{NS}}$. The latter seems to be a more natural choice here since $M_{S}$ is related to a potentially small Yukawa coupling and a symmetry breaking. ${ }^{1}$

There is one important thing we would like to point out here. In our model we have basically only one mass scale which is the SUSY breaking scale (assuming that the $\mu$ parameters are of the same order). This has to be seen in contrast to the conventional seesaw models where there is another superheavy seesaw scale besides the electroweak scale. Hence, in our model the question what triggers these huge gap between the two scales simply does not occur.

The original definition of the inverse seesaw mechanism implies $M_{S} \ll M_{D} \ll \mu_{\mathrm{NS}}$. Here we generalize this definition to realize three different types of the inverse seesaw mechanisms according to the assumed hierarchies in the masses

(i) ISS type I: $M_{S} \ll M_{D} \ll \mu_{\mathrm{NS}}$,

(ii) ISS type II: $M_{S} \sim M_{D} \ll \mu_{\mathrm{NS}}$,

(iii) ISS type III: $M_{D} \ll M_{S} \ll \mu_{\mathrm{NS}}$.

The different cases are in the end assumptions about the size of the involved Yukawa couplings. Keep in mind that the electroweak and $Z_{6}$ symmetry breakings are related to soft SUSY breaking parameters and it is plausible to assume that the vevs are similar in size. The sizes of the Yukawa couplings are here not as well motivated and in the following we discuss the three cases mentioned above. Note that these are simplified assumptions though. In reality, it could well be that one generation looks more like ISS type I while another generation behaves like type III.

One big advantage of this simplified assumption is that we can do a proper expansion of the neutrino mass and mixing in terms of some expansion parameters, which we discuss soon for the three cases mentioned above. Without loss of generality we also choose a basis where $\mu_{\mathrm{NS}}$ is diagonal, which implies in particular that $\mu_{\mathrm{NS}}^{T}=\mu_{\mathrm{NS}}$ from now on unless stated otherwise.

\footnotetext{
${ }^{1}$ From that point of view an inverse seesaw mechanism is technically natural a la 't-Hooft [38].
} 
Before we go through the details of the different types we would like to anticipate one common result: in all three cases, the leading order expression for the light neutrino mass matrix is the same and given by

$$
m_{\nu}=M_{D} \mu_{\mathrm{NS}}^{-1} M_{S} \mu_{\mathrm{NS}}^{-1} M_{D}^{T},
$$

which is nothing else than the ordinary inverse seesaw formula and the other heavier mass eigenstates have masses of the order of $\mu_{\mathrm{NS}} \sim \mathrm{TeV}$ with small corrections. From the above formula it is also obvious that the inverse seesaw mechanism is in our model a direct consequence of the $Z_{6}$ breaking $\left(M_{S} \sim v_{X}\right)$.

The above formula can be rewritten

$$
m_{\nu}=Y_{\nu} \mu_{\mathrm{NS}}^{-1} \lambda \mu_{\mathrm{NS}}^{-1} Y_{\nu}^{T} v_{u}^{2} v_{X} \sim Y_{\nu} \lambda Y_{\nu}^{T} \mathcal{O}(\mathrm{TeV}),
$$

where we have used the working assumption that the dimensionful quantities $v_{u}, v_{X}$ and $\mu_{\mathrm{NS}}$ are all of the same order. The smallness of neutrino masses is hence completely given by the moderate smallness of the Yukawa couplings $Y_{\nu}$ and $\lambda$. Their size is related to the size of the respective expansion parameter as we will discuss in the following for the three different ISS cases.

\subsubsection{ISS type I}

In this case we assume that $\mu_{\mathrm{NS}}$ is $\mathcal{O}(\mathrm{TeV}), M_{D} \sim \epsilon_{\mathrm{I}} \mu_{\mathrm{NS}}$ and $M_{S} \sim \epsilon_{\mathrm{I}}^{2} \mu_{\mathrm{NS}}$ where $\epsilon_{\mathrm{I}}$ is the expansion parameter. We will quote the size of $\epsilon_{\mathrm{I}}$ at the end of this subsection after deriving the expression for the light neutrino masses.

Note that we start with the product $M_{\nu} M_{\nu}^{\dagger}$ instead of $M_{\nu}$ alone. We diagonalise the matrix $M_{\nu} M_{\nu}^{\dagger}$ in two steps. First, we do a block rotation, $W$, to separate the light from the heavy states sufficiently involving only small mixing angles. Then we are left with another rotation $V$, which acts upon the light and the heavy states separately. In particular the rotation for the light states is the PMNS matrix to a good approximation. So our diagonalisation condition reads

$$
\begin{aligned}
U_{\mathrm{I}} M_{\nu} M_{\nu}^{\dagger} U_{\mathrm{I}}^{\dagger} & =V_{\mathrm{I}} W_{\mathrm{I}} M_{\nu} M_{\nu}^{\dagger} W_{\mathrm{I}}^{\dagger} V_{\mathrm{I}}^{\dagger}=V_{\mathrm{I}}\left(\begin{array}{cc}
m_{\nu} m_{\nu}^{\dagger} & \mathcal{O}\left(\epsilon_{\mathrm{I}}^{7}\right) \\
\mathcal{O}\left(\epsilon_{\mathrm{I}}^{7}\right) & M_{R} M_{R}^{\dagger}
\end{array}\right) V_{\mathrm{I}}^{\dagger} \\
& =\left(\begin{array}{cc}
U_{\mathrm{PMNS}} & 0 \\
0 & R_{\mathrm{I}}
\end{array}\right)\left(\begin{array}{cc}
m_{\nu} m_{\nu}^{\dagger} & \mathcal{O}\left(\epsilon_{\mathrm{I}}^{7}\right) \\
\mathcal{O}\left(\epsilon_{\mathrm{I}}^{7}\right) & M_{R} M_{R}^{\dagger}
\end{array}\right)\left(\begin{array}{ccc}
U_{\mathrm{PMNS}}^{\dagger} & 0 \\
0 & R_{\mathrm{I}}^{\dagger}
\end{array}\right),
\end{aligned}
$$

where $U_{\mathrm{PMNS}}$ and $R_{\mathrm{I}}$ diagonalise only the upper $3 \times 3$ and the lower $4 \times 4$ blocks, respectively. As we will see very soon $m_{\nu} m_{\nu}^{\dagger}$ is of $\mathcal{O}\left(\epsilon_{\mathrm{I}}^{8}\right)$ and $M_{R} M_{R}^{\dagger}$ is of $\mathcal{O}(1)$. Hence, the remaining off-diagonal elements of $\mathcal{O}\left(\epsilon_{\mathrm{I}}^{7}\right)$ are negligible.

We present an explicit expression for $W_{\mathrm{I}}$ and its elements $w_{i j}$ in appendix A. Here we just present $W_{\mathrm{I}}$ and $U_{\mathrm{I}}$ in terms of the leading order in $\epsilon_{\mathrm{I}}$

$$
W_{\mathrm{I}} \sim\left(\begin{array}{ccc}
1 & w_{12} \eta_{\mathrm{I}}^{3} & w_{13} \eta_{\mathrm{I}} \\
w_{21} \eta_{\mathrm{I}}^{3} & 1 & w_{23} \eta_{\mathrm{I}}^{8} \\
w_{31} \eta_{\mathrm{I}} & w_{32} \eta_{\mathrm{I}}^{4} & 1
\end{array}\right) \text { and } U_{\mathrm{I}} \sim\left(\begin{array}{cc}
U_{\mathrm{PMNS}} & \eta_{\mathrm{I}}^{3} U_{\mathrm{PMNS}} w_{12} \eta_{\mathrm{I}} U_{\mathrm{PMNS}} w_{13} \\
R_{\mathrm{I}}\left(\begin{array}{c}
w_{21} \eta_{\mathrm{I}}^{3} \\
w_{31} \eta_{\mathrm{I}}
\end{array}\right) & R_{\mathrm{I}}
\end{array}\right)
$$


We have introduced here $\eta_{\mathrm{I}}=1$ which labels the order of the matrix elements in $\epsilon_{\mathrm{I}}$. For instance, we write $w_{12} \eta_{\mathrm{I}}^{3}$ which states that the element $w_{12}$ is $\mathcal{O}\left(\epsilon_{\mathrm{I}}^{3}\right)$.

For the light and heavy mass matrices we only quote the leading and next-to-leading order contributions

$$
\begin{aligned}
m_{\nu} m_{\nu}^{\dagger}= & \eta_{\mathrm{I}}^{8} M_{D} \mu_{\mathrm{NS}}^{-1} M_{S} \mu_{\mathrm{NS}}^{-1} M_{D}^{T} M_{D}^{*}\left(\mu_{\mathrm{NS}}^{*}\right)^{-1} M_{S}^{*}\left(\mu_{\mathrm{NS}}^{*}\right)^{-1} M_{D}^{\dagger} \\
& -\frac{1}{2} \eta_{\mathrm{I}}^{10} M_{D}\left(\mu_{\mathrm{NS}}\right)^{-1}\left(M_{S}\left(\mu_{\mathrm{NS}}\right)^{-1} M_{D}^{T} M_{D}^{*}\left(\mu_{\mathrm{NS}}^{*}\right)^{-1} M_{S}^{*}\left(\mu_{\mathrm{NS}}^{*}\right)^{-1} M_{D}^{\dagger} M_{D}\left(\mu_{\mathrm{NS}}\right)^{-1}\right. \\
& +2 M_{S}\left(\mu_{\mathrm{NS}}\right)^{-1} M_{D}^{T} M_{D}^{*}\left(\mu_{\mathrm{NS}}^{*}\right)^{-1}\left(\mu_{\mathrm{NS}}\right)^{-1} M_{D}^{T} M_{D}^{*}\left(\mu_{\mathrm{NS}}^{*}\right)^{-1} M_{S}^{*} \\
& \left.+\left(\mu_{\mathrm{NS}}^{*}\right)^{-1} M_{D}^{\dagger} M_{D}\left(\mu_{\mathrm{NS}}\right)^{-1} M_{S}\left(\mu_{\mathrm{NS}}\right)^{-1} M_{D}^{T} M_{D}^{*}\left(\mu_{\mathrm{NS}}^{*}\right)^{-1} M_{S}^{*}\right)\left(\mu_{\mathrm{NS}}^{*}\right)^{-1} M_{D}^{\dagger} \\
M_{R} M_{R}^{\dagger}= & \left(\begin{array}{cc}
\mu_{\mathrm{NS}} \mu_{\mathrm{NS}}^{*}+\eta_{\mathrm{I}}^{2} M_{D}^{T} M_{D}^{*} \\
\eta_{\mathrm{I}}^{2} M_{S} \mu_{\mathrm{NS}}^{*} \mu_{\mathrm{NS}} M_{S}^{*}
\end{array}\right)
\end{aligned}
$$

where we have quoted for convenience the orders in $\epsilon_{\mathrm{I}}$ explicitly using $\eta_{\mathrm{I}}$.

In our minimal setup $M_{D}$ is a $3 \times 2$ matrix and therefore the lightest neutrino is strictly massless due to rank considerations. Our neutrino mass scale is hence given by $\sqrt{\Delta m_{32}^{2}} \approx$ $5 \cdot 10^{-2} \mathrm{eV}$ and $\epsilon_{\mathrm{I}} \sim(0.01 \mathrm{eV} / \mathrm{TeV})^{1 / 4} \sim 10^{-4}$. This implies that $Y_{\nu} \sim 10^{-4}$ and $\lambda \sim 10^{-8}$.

\subsubsection{ISS type II}

In the ISS type II we have again that $\mu_{\mathrm{NS}}$ is $\mathcal{O}(\mathrm{TeV})$ but now $M_{D} \sim M_{S} \sim \epsilon_{\mathrm{II}} \mu_{\mathrm{NS}}$. Our diagonalisation reads now

$$
\begin{aligned}
U_{\mathrm{II}} M_{\nu} M_{\nu}^{\dagger} U_{\mathrm{II}}^{\dagger} & =V_{\mathrm{II}} W_{\mathrm{II}} M_{\nu} M_{\nu}^{\dagger} W_{\mathrm{II}}^{\dagger} V_{\mathrm{II}}^{\dagger}=V_{\mathrm{II}}\left(\begin{array}{cc}
m_{\nu} m_{\nu}^{\dagger} & \mathcal{O}\left(\epsilon_{\mathrm{II}}^{5}\right) \\
\mathcal{O}\left(\epsilon_{\mathrm{II}}^{5}\right) & M_{R} M_{R}^{\dagger}
\end{array}\right) V_{\mathrm{II}}^{\dagger} \\
& =\left(\begin{array}{cc}
U_{\mathrm{PMNS}} & 0 \\
0 & R_{\mathrm{II}}
\end{array}\right)\left(\begin{array}{cc}
m_{\nu} m_{\nu}^{\dagger} & \mathcal{O}\left(\epsilon_{\mathrm{II}}^{5}\right) \\
\mathcal{O}\left(\epsilon_{\mathrm{II}}^{5}\right) & M_{R} M_{R}^{\dagger}
\end{array}\right)\left(\begin{array}{cc}
U_{\mathrm{PMNS}}^{\dagger} & 0 \\
0 & R_{\mathrm{II}}^{\dagger}
\end{array}\right) .
\end{aligned}
$$

The neutrino mass matrices are

$$
\begin{aligned}
m_{\nu} m_{\nu}^{\dagger}= & \eta_{\mathrm{II}}^{6} M_{D} \mu_{\mathrm{NS}}^{-1} M_{S} \mu_{\mathrm{NS}}^{-1} M_{D}^{T} M_{D}^{*}\left(\mu_{\mathrm{NS}}^{*}\right)^{-1} M_{S}^{*}\left(\mu_{\mathrm{NS}}^{*}\right)^{-1} M_{D}^{\dagger} \\
& -\frac{1}{2} \eta_{\mathrm{II}}^{8} M_{D} \mu_{\mathrm{NS}}^{-1} M_{S} \mu_{\mathrm{NS}}^{-1} M_{D}^{T} M_{D}^{*}\left(\mu_{\mathrm{NS}}^{*}\right)^{-1} M_{S}^{*}\left(\mu_{\mathrm{NS}}^{*}\right)^{-1} M_{D}^{\dagger} M_{D} \mu_{\mathrm{NS}}^{-1}\left(\mu_{\mathrm{NS}}^{*}\right)^{-1} M_{D}^{\dagger} \\
& -\eta_{\mathrm{II}}^{8} M_{D} \mu_{\mathrm{NS}}^{-1} M_{S} \mu_{\mathrm{NS}}^{-1} M_{D}^{T} M_{D}^{*}\left(\mu_{\mathrm{NS}}^{*}\right)^{-1} \mu_{\mathrm{NS}}^{-1} M_{D}^{T} M_{D}^{*}\left(\mu_{\mathrm{NS}}^{*}\right)^{-1} M_{S}^{*}\left(\mu_{\mathrm{NS}}^{*}\right)^{-1} M_{D}^{\dagger} \\
& -\frac{1}{2} \eta_{\mathrm{II}}^{8} M_{D} \mu_{\mathrm{NS}}^{-1}\left(\mu_{\mathrm{NS}}^{*}\right)^{-1} M_{D}^{\dagger} M_{D} \mu_{\mathrm{NS}}^{-1} M_{S} \mu_{\mathrm{NS}}^{-1} M_{D}^{T} M_{D}^{*}\left(\mu_{\mathrm{NS}}^{*}\right)^{-1} M_{S}^{*}\left(\mu_{\mathrm{NS}}^{*}\right)^{-1} M_{D}^{\dagger}, \\
\left(M_{R} M_{R}^{\dagger}\right)_{11} & =\mu_{\mathrm{NS}} \mu_{\mathrm{NS}}^{*}+\eta_{\mathrm{II}}^{2} M_{D}^{T} M_{D}^{*} \\
\left(M_{R} M_{R}^{\dagger}\right)_{22} & =\mu_{\mathrm{NS}} \mu_{\mathrm{NS}}^{*}+\eta_{\mathrm{II}}^{2} M_{S} M_{S}^{*}+1 / 2 \eta_{\mathrm{II}}^{2} \mu_{\mathrm{NS}} M_{D}^{\dagger} M_{D} \mu_{\mathrm{NS}}^{-1} \\
& +1 / 2 \eta_{\mathrm{II}}^{2}\left(\mu_{\mathrm{NS}}^{*}\right)^{-1} M_{D}^{\dagger} M_{D} \mu_{\mathrm{NS}}^{*} \\
\left(M_{R} M_{R}^{\dagger}\right)_{12} & =\eta_{\mathrm{II}} \mu_{\mathrm{NS}} M_{S}^{*} \\
\left(M_{R} M_{R}^{\dagger}\right)_{21} & =\eta_{\mathrm{II}} M_{S} \mu_{\mathrm{NS}}^{*} .
\end{aligned}
$$


For later reference we write down explicitly $W_{\text {II }}$ and $U_{\text {II }}$ up to the leading orders in $\epsilon_{\text {II }}$

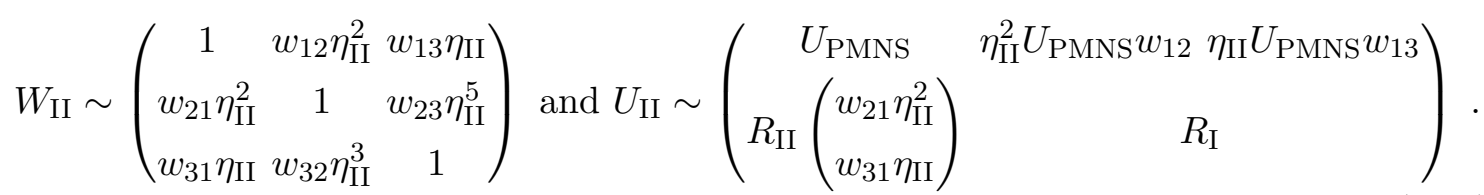

We have introduced here $\eta_{\mathrm{II}}$ to label the order of the elements in $\epsilon_{\mathrm{II}}$ for convenience similar to ISS type I. The explicit expression for $W_{\text {II }}$ can be found in appendix A.

For the expansion parameter $\epsilon_{\mathrm{II}}$ in ISS type II we find $\epsilon_{\mathrm{II}} \sim(0.01 \mathrm{eV} / \mathrm{TeV})^{1 / 3} \sim 10^{-5}$ which is one order smaller than in ISS type I. For the Yukawa couplings this implies $Y_{\nu} \sim \lambda \sim 10^{-5}$.

\subsubsection{ISS type III}

In the ISS type III we have again that $\mu_{\mathrm{NS}}$ is $\mathcal{O}(\mathrm{TeV})$ but now $M_{S} \sim \epsilon_{\mathrm{III}} \mu_{\mathrm{NS}}$ and $M_{D} \sim$ $\epsilon_{\mathrm{III}}^{2} \mu_{\mathrm{NS}}$. Our diagonalisation reads here

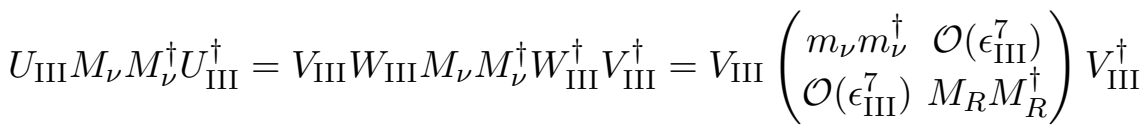

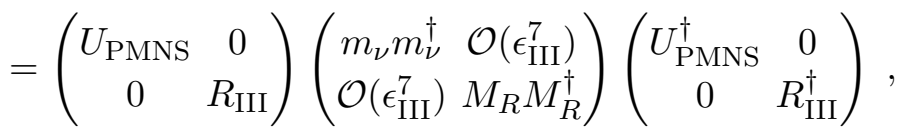

The explicit expression for $W_{\text {III }}$ can be found in appendix A. The neutrino mass matrices are

$$
\begin{aligned}
m_{\nu} m_{\nu}^{\dagger} & =\eta_{\mathrm{III}}^{10} M_{D} \mu_{\mathrm{NS}}^{-1} M_{S} \mu_{\mathrm{NS}}^{-1} M_{D}^{T} M_{D}^{*}\left(\mu_{\mathrm{NS}}^{*}\right)^{-1} M_{S}^{*}\left(\mu_{\mathrm{NS}}^{*}\right)^{-1} M_{D}^{\dagger}+\mathcal{O}\left(\eta_{\mathrm{III}}^{14}\right) \\
M_{R} M_{R}^{\dagger} & =\left(\begin{array}{cc}
\mu_{\mathrm{NS}} \mu_{\mathrm{NS}}^{*} & \eta_{\mathrm{III}} \mu_{\mathrm{NS}} M_{S}^{*} \\
\eta_{\mathrm{III}} M_{S} \mu_{\mathrm{NS}}^{*} & \mu_{\mathrm{NS}} \mu_{\mathrm{NS}}^{*}+\eta_{\mathrm{III}}^{2} M_{S} M_{S}^{*}
\end{array}\right)
\end{aligned}
$$

For later reference we write down explicitly the leading orders of $W_{\text {III }}$ and $U_{\text {III }}$

$$
\begin{aligned}
W_{\mathrm{III}} \sim\left(\begin{array}{ccc}
1 & w_{12} \eta_{\mathrm{III}}^{3} & w_{13} \eta_{\mathrm{III}}^{2} \\
w_{21} \eta_{\mathrm{III}}^{3} & 1 & \mathcal{O}\left(\eta_{\mathrm{III}}^{13}\right) \\
w_{31} \eta_{\mathrm{III}}^{2} & w_{32} \eta_{\mathrm{III}}^{5} & 1
\end{array}\right) \text { and } \\
U_{\mathrm{III}} \sim\left(\begin{array}{cc}
U_{\mathrm{PMNS}} & \eta_{\mathrm{III}}^{3} U_{\mathrm{PMNS}} w_{12} \eta_{\mathrm{III}}^{2} U_{\mathrm{PMNS}} w_{13} \\
R_{\mathrm{III}}\left(\begin{array}{l}
w_{21} \eta_{\mathrm{III}}^{3} \\
w_{31} \eta_{\mathrm{III}}^{2}
\end{array}\right) & R_{\mathrm{III}}
\end{array}\right) .
\end{aligned}
$$

We have introduced here $\eta_{\mathrm{III}}$ to label the order of the elements in $\epsilon_{\mathrm{III}}$ for convenience similar to ISS type I. The explicit expression for $W_{\text {III }}$ can be found in appendix A.

For the expansion parameter in ISS type III we find $\epsilon_{\mathrm{III}} \sim(0.01 \mathrm{eV} / \mathrm{TeV})^{1 / 5} \sim 10^{-3}$. ISS type III exhibits hence the mildest hierarchies and it has the smallest neutrino Yukawa couplings, $Y_{\nu} \sim 10^{-6}$, and the largest singlet Yukawa coupling, $\lambda \sim 10^{-3}$. 
It is also remarkable that in all three cases the leading order formulas for the light and the heavy neutrino masses are the same but there are differences in the next-toleading order terms which might potentially help to disentangle the three cases in precision measurements in the future.

\subsubsection{Non-unitarity of the mixing matrix}

At this point we would like to comment on the non-unitarity of the PMNS matrix obtained in our formalism. Only the full $7 \times 7$ mixing matrix will be unitary while any given submatrix of this matrix does not have to be unitary. Let us illustrate this first with the ISS type I as an example

$$
\begin{aligned}
& 1=U_{\mathrm{I}} U_{\mathrm{I}}^{\dagger}=\left(\begin{array}{cc}
U_{\mathrm{PMNS}} & 0 \\
0 & R_{\mathrm{I}}
\end{array}\right) W_{\mathrm{I}} W_{\mathrm{I}}^{\dagger}\left(\begin{array}{cc}
U_{\mathrm{PMNS}}^{\dagger} & 0 \\
0 & R_{\mathrm{I}}^{\dagger}
\end{array}\right)
\end{aligned}
$$

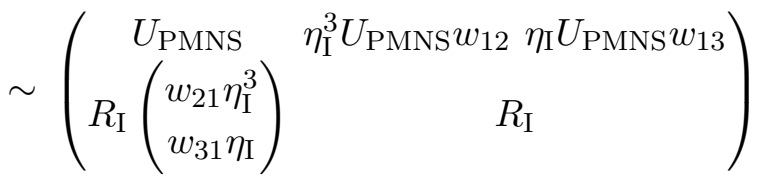

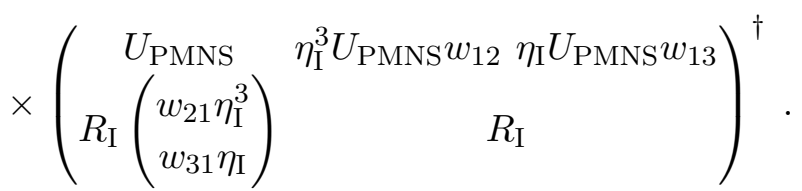

For simplicity, we consider now only the first $3 \times 3$ block up to $\mathcal{O}\left(\epsilon_{\mathrm{I}}^{2}\right)$ which we are interested in

$$
1 \approx U_{\mathrm{PMNS}} U_{\mathrm{PMNS}}^{\dagger}+\eta_{\mathrm{I}}^{2} U_{\mathrm{PMNS}} w_{13} w_{13}^{\dagger} U_{\mathrm{PMNS}}^{\dagger}
$$

Note that expanding in $\epsilon_{\mathrm{I}}$ or $\eta_{\mathrm{I}}$ gives here the same results since they always appear together at the same order. Of course, technically speaking we have to expand in $\epsilon_{\mathrm{I}}$ since this is the small parameter while $\eta_{\mathrm{I}}$ is only a bookkeeping parameter equal to one (and not small). After multiplying this equation from left with $U_{\text {PMNS }}^{-1}$, from right with $\left(U_{\text {PMNS }}^{\dagger}\right)^{-1}$ and inverting the whole equation we find

$$
U_{\mathrm{PMNS}}^{\dagger} U_{\mathrm{PMNS}} \approx\left(1+\eta_{\mathrm{I}}^{2} w_{13} w_{13}^{\dagger}\right)^{-1}
$$

so that the deviation from unitarity is of $\mathcal{O}\left(\epsilon_{\mathrm{I}}^{2}\right)=\mathcal{O}\left(10^{-8}\right)$, which is much smaller than current constraints, see, for example, [39], but might be relevant in the future.

For the other two ISS types we find even smaller deviations from unitarity of $\mathcal{O}\left(\epsilon_{\mathrm{II}}^{2}\right)=$ $\mathcal{O}\left(10^{-10}\right)$ and $\mathcal{O}\left(\epsilon_{\mathrm{III}}^{4}\right)=\mathcal{O}\left(10^{-12}\right)$, respectively.

\subsubsection{The Yukawa couplings}

Although at this point we do not need it explicitly we derive some expressions for the Dirac neutrino Yukawa coupling constants in terms of the Casas-Ibarra parameterization [40]. The advantage is that after fixing unknown parameters we can immediately calculate the Yukawa matrix such that neutrino oscillation data is correctly reproduced in our model. 
From the neutrino mass matrix eq. (3.6), the leading contribution to active neutrino masses is obtained as

$$
m_{i} \equiv U_{\mathrm{PMNS}}^{\dagger} m_{\nu} U_{\mathrm{PMNS}}^{*}=U_{\mathrm{PMNS}}^{\dagger} M_{D} \mu_{\mathrm{NS}}^{-1} M_{S}\left(\mu_{\mathrm{NS}}^{-1}\right)^{T} M_{D}^{T} U_{\mathrm{PMNS}}^{*}
$$

where $m_{i}$ is the diagonal mass matrix of light, active neutrino states, $m_{i}=$ $\operatorname{diag}\left(m_{1}, m_{2}, m_{3}\right)$. Note that we have transposed here the second $\mu_{\mathrm{NS}}^{-1}$ for later convenience, which we have not before since we are working in a basis where $\mu_{\mathrm{NS}}$ is diagonal.

Since $M_{S}$ is not diagonal in general, we first need to diagonalise this matrix by a unitary matrix $V_{S}, M_{S}^{d} \equiv V_{S} M_{S} V_{S}^{T}$. We can use this in eq. (3.26) and find

$$
\sqrt{m_{i}} \sqrt{m_{i}}=U_{\mathrm{PMNS}}^{\dagger} M_{D} \mu_{\mathrm{NS}}^{-1} V_{S}^{\dagger} \sqrt{M_{S}^{d}} \sqrt{M_{S}^{d}} V_{S}^{*}\left(\mu_{\mathrm{NS}}^{-1}\right)^{T} M_{D}^{T} U_{\mathrm{PMNS}}^{*}
$$

from where we can easily derive the leading order expression for the neutrino Yukawa couplings

$$
Y_{\nu}=\frac{\mathrm{i}}{v_{u}} U_{\mathrm{PMNS}} \sqrt{m_{i}} \Omega\left(\sqrt{M_{S}^{d}}\right)^{-1} V_{S} \mu_{\mathrm{NS}}
$$

where $\Omega$ is an arbitrary, orthogonal, complex matrix parameterized by

$$
\Omega^{\mathrm{NH}}=\left(\begin{array}{cc}
0 & 0 \\
\cos \omega & \sin \omega \\
-\xi \sin \omega & \xi \cos \omega
\end{array}\right), \quad \Omega^{\mathrm{IH}}=\left(\begin{array}{cc}
\cos \omega & \sin \omega \\
-\xi \sin \omega & \xi \cos \omega \\
0 & 0
\end{array}\right)
$$

with $\omega$ being a complex parameter and $\xi= \pm 1$ corresponding to a parity degree of freedom. Here NH denotes normal neutrino mass hierarchy and IH denotes inverted neutrino mass hierarchy.

\subsection{Neutrinoless double beta decay}

Once massive Majorana neutrinos are implemented into the SM, global lepton number symmetry is broken by two units and an interesting phenomenom called neutrinoless double beta $(0 \nu \beta \beta)$ decay can occur, for a recent review see [41]. The rate of $0 \nu \beta \beta$ decay is proportional to the modulus square of the effective mass, $m_{\mathrm{eff}}$, which is gradually constrained by several experiments. The most stringent bound so far, $\left|m_{\mathrm{eff}}\right|<(61-161) \mathrm{meV}$, is from the search for $0 \nu \beta \beta$ decay of ${ }^{136} \mathrm{Xe}$ by the KamLAND-Zen collaboration [42].

When we introduce only three massive Majorana neutrinos, $m_{\mathrm{eff}}$ can be expressed as

$$
m_{\mathrm{eff}}^{\text {active }}=\sum_{i=1}^{3}\left(U_{\mathrm{PMNS}}\right)_{e i}^{2} m_{i}
$$

where $m_{i}$ are the mass eigenvalues of the neutrinos and $m_{1}\left(m_{3}\right)$ is exactly equal to zero in our model for the NH (IH) case. When we take the active neutrino mass and the mixing angles given in [43] we obtain that $\left|m_{\text {eff }}\right|$ is $\mathcal{O}(1)$ and $\mathcal{O}(10) \mathrm{meV}$ in the $\mathrm{NH}$ and IH cases, respectively. In addition to this standard contribution coming from the active neutrinos, 
one can get some other contributions in extensions of the minimal model with three light active neutrinos only.

Especially, as we have mentioned before, there is no $R$-parity in our model, and one might think of some contributions from the exchange of SUSY particles. The SUSY contributions of $0 \nu \beta \beta$ decay are induced by the $R$-parity violating $\hat{L} \hat{Q} \hat{D}^{c}$ interaction of the first generation [44]. However, such a term is forbidden due to the $Z_{6}$ symmetry imposed in our model. It means that we do not have any SUSY contribution to $0 \nu \beta \beta$ decay.

As a result, we can focus on the non-SUSY contributions of the model. The contributions can be parameterized as

$$
m_{\mathrm{eff}}^{\mathrm{new}}=\sum_{i=4}^{7}\left(U_{e i}\right)^{2} m_{i} f_{\beta}\left(m_{i}\right),
$$

where $f_{\beta}(x)$ denotes the suppression factor of the nuclear matrix element when the mass scale $x$ is larger than a typical scale $\mathcal{O}(100 \mathrm{MeV})$. Since the typical mass scales for additional gauge singlet fermions is $\mu_{\mathrm{NS}}$ as we discussed above, we simply replace $x$ by $x=\mu_{\mathrm{NS}}$ and treat $\mu_{\mathrm{NS}}$ like a number for simplicity throughout the rest of this section. In the current analysis, we adopt the expression

$$
f_{\beta}\left(\mu_{\mathrm{NS}}\right)=\frac{\left\langle p^{2}\right\rangle}{\mu_{\mathrm{NS}}^{2}+\left\langle p^{2}\right\rangle},
$$

with the typical momentum in the matrix element $\left\langle p^{2}\right\rangle \simeq(200 \mathrm{MeV})^{2}$ [45]. As the typical mass scale of the heavy neutrinos is of $\mathcal{O}(\mathrm{TeV})$, their contribution is given by $[36,46]$

$$
\begin{aligned}
m_{\mathrm{eff}}^{\text {new }} & \simeq \sum_{i=4}^{7}\left(U_{e i}\right)^{2} \frac{\left\langle p^{2}\right\rangle}{\mu_{\mathrm{NS}}^{2}} m_{i} \\
& =\left\langle p^{2}\right\rangle\left[-\left(U_{e 4}\right)^{2} \frac{\left|m_{4}\right|}{\mu_{\mathrm{NS}}^{2}}+\left(U_{e 5}\right)^{2} \frac{\left|m_{5}\right|}{\mu_{\mathrm{NS}}^{2}}-\left(U_{e 6}\right)^{2} \frac{\left|m_{6}\right|}{\mu_{\mathrm{NS}}^{2}}+\left(U_{e 7}\right)^{2} \frac{\left|m_{7}\right|}{\mu_{\mathrm{NS}}^{2}}\right] .
\end{aligned}
$$

Due to the particular structure of the mass matrix there are always two mass eigenstates with almost the same mass but opposite sign as suggested in the above formula. To be more precise all the absolute values of the heavier masses are given at the leading order by $\mu_{\mathrm{NS}}$. At this order the cancellation is exact since also $\left(U_{e 4}\right)^{2}=\left(U_{e 5}\right)^{2}$ and $\left(U_{e 6}\right)^{2}=\left(U_{e 7}\right)^{2}$. Nevertheless, this cancellation is not exact to all orders and the first non-vanishing order in ISS type I is obtained as

$$
m_{\mathrm{eff}}^{\text {new }} \simeq \sum_{i=4}^{7}\left(U_{e i}\right)^{2} \frac{\left\langle p^{2}\right\rangle}{\mu_{\mathrm{NS}}^{2}} M_{S} \lesssim \epsilon_{\mathrm{I}}^{4} \cdot\left(8 \times 10^{7} \mathrm{meV}\right) \cdot\left(\frac{\mathrm{TeV}}{\mu_{\mathrm{NS}}}\right) \approx 8 \times 10^{-9} \mathrm{meV} \cdot\left(\frac{\mathrm{TeV}}{\mu_{\mathrm{NS}}}\right),
$$

which is negligibly small compared to the contribution from the light active neutrinos. In ISS type II and III the contributions are even smaller as can be easily checked.

\subsection{Charged lepton flavor violation}

In the SM, cLFV is not allowed on the perturbative level, but this will immediately change once neutrino masses are introduced. In the following we discuss some estimates for cLFV in our model. 


\subsubsection{The non-SUSY part}

Let us begin with the discussion on the non-SUSY part which corresponds to sending the SUSY breaking scale to infinity and there are no contributions from the SUSY partners to the process $\mu \rightarrow e \gamma$ for instance. To estimate the contributions of these processes we refer to an early calculation by Cheng and Li [47], see also [48], but adapt their notation to our conventions. We quote for simplicity the formulas for $\mu \rightarrow e \gamma$ only. The expressions can be straight-forwardly extended to other processes. Each neutrino-like mass eigenstate contributes to the amplitude

$$
A_{i}=\frac{G_{F}}{\sqrt{2}} \frac{e m_{\mu}}{32 \pi^{2}} U_{i e} U_{i \mu}^{*} F\left(m_{i}^{2} / M_{W}^{2}\right),
$$

where $i=1, \ldots, 7$ and

$$
F(x)=\frac{10-43 x+78 x^{2}-49 x^{3}+4 x^{4}+18 x^{3} \log x}{3(x-1)^{4}}
$$

for $x>0$ and $x \neq 1$. For $x \ll 1$ this simplifies to

$$
F(x)=\frac{10}{3}-x+\mathcal{O}\left(x^{2}\right)
$$

and for $x \gg 1$

$$
F(x)=\frac{4}{3}-\frac{1}{x}\left(11+6 \log \frac{1}{x}\right)+\mathcal{O}\left(x^{-2}\right) .
$$

The physical branching ratio (BR) is $\propto\left|\sum_{i} A_{i}\right|^{2}$. So we shall first identify the largest amplitude $A_{i}$ to get a feeling for the maximal $\mathrm{BR}$ we can expect. The neutrino-like mass eigenstates are either much lighter or much heavier than the $W$-boson, $x \ll 1$ or $1 / x \ll 1$. Therefore, the dominant contribution is coming from the constant term of $F(x)$ and we consider the two cases separately. Let us begin with the light states, $i=1,2,3$. If there would be only three light states which do not mix with any other states we would find $\sum_{i=1}^{3} U_{i e} U_{i \mu}^{*}=0$ due to the unitarity of the PMNS matrix. Therefore, the leading term contributions proportional to the constant term in eq. (3.37) all cancel out. The next leading term in eq. (3.37) would be $x=m_{\nu}^{2} / M_{W}^{2} \sim 10^{-20}$, which is negligible compared with the incomplete unitarity that we discuss now. The unitarity is only complete when summed over all $i=1-7$, see the discussion in section 3.1.4, and therefore

$$
\sum_{i=1}^{3} U_{i e} U_{i \mu}^{*}= \begin{cases}\mathcal{O}\left(\epsilon_{\mathrm{I}}^{2}\right)=\mathcal{O}\left(10^{-8}\right) & \text { for ISS type I } \\ \mathcal{O}\left(\epsilon_{\mathrm{II}}^{2}\right)=\mathcal{O}\left(10^{-10}\right) & \text { for ISS type II } \\ \mathcal{O}\left(\epsilon_{\mathrm{III}}^{4}\right)=\mathcal{O}\left(10^{-12}\right) & \text { for ISS type III. }\end{cases}
$$

Since $U$ as a $7 \times 7$ matrix is unitary this non-vanishing has to be compensated by the heavy states such that we find as well

$$
\sum_{i=4}^{7} U_{i e} U_{i \mu}^{*}= \begin{cases}\mathcal{O}\left(\epsilon_{\mathrm{I}}^{2}\right)=\mathcal{O}\left(10^{-8}\right) & \text { for ISS type I, } \\ \mathcal{O}\left(\epsilon_{\mathrm{II}}^{2}\right)=\mathcal{O}\left(10^{-10}\right) & \text { for ISS type II } \\ \mathcal{O}\left(\epsilon_{\mathrm{III}}^{4}\right)=\mathcal{O}\left(10^{-12}\right) & \text { for ISS type III. }\end{cases}
$$




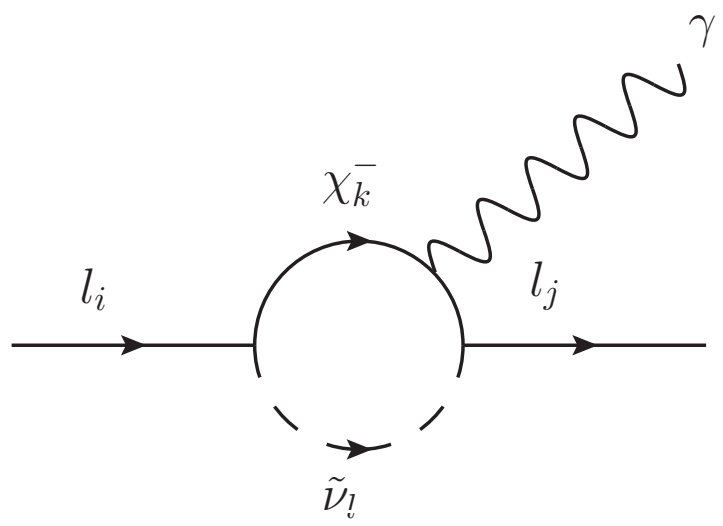

Figure 1. Feynman diagram for charged lepton flavor violation which changes compared to the case of the MSSM extended by right-handed neutrinos.

The branching ratio is defined with respect to the width of the muon $\Gamma(\mu \rightarrow e \nu \bar{\nu})=$ $m_{\mu}^{5} G_{F}^{2} / 192 \pi^{3}$ such that we find

$$
\begin{aligned}
\operatorname{BR}(\mu \rightarrow e \gamma) & =\frac{48 \pi^{2}\left|\sum_{i} A_{i}\right|^{2}}{m_{\mu}^{2} G_{F}^{2}}=\frac{3 \alpha}{32 \pi}\left|\sum_{i} U_{i e} U_{i \mu}^{*} F\left(m_{i}^{2} / M_{W}^{2}\right)\right|^{2} \\
& = \begin{cases}\mathcal{O}\left(10^{-20}\right) & \text { for ISS type I, } \\
\mathcal{O}\left(10^{-24}\right) & \text { for ISS type II, } \\
\mathcal{O}\left(10^{-28}\right) & \text { for ISS type III, }\end{cases}
\end{aligned}
$$

which are all far below the current bound $\operatorname{BR}(\mu \rightarrow e \gamma)<4.2 \times 10^{-13}$ at $90 \%$ confidence level of the MEG experiment [49]. The branching ratios for other cLFV processes are similarly suppressed but their bounds are generally weaker.

\subsubsection{The SUSY part}

There are also contributions to cLFV from loops involving supersymmetric partners [50, 51]. While the pieces involving the charged sleptons do not change, there are major changes for the contributions involving scalar partners of the neutrinos (of both chiralities) and the singlets, cf. figure 1. Importantly, $X$ receives a vev which induces a mass splitting for the $\mathrm{CP}$-even and $\mathrm{CP}$-odd components of the sneutrinos and we define

$$
\begin{aligned}
\tilde{\nu}_{L} & =\frac{1}{\sqrt{2}}\left(\phi_{L}+\mathrm{i} \sigma_{L}\right), \\
\tilde{N} & =\frac{1}{\sqrt{2}}\left(\phi_{R}+\mathrm{i} \sigma_{R}\right), \\
\tilde{S} & =\frac{1}{\sqrt{2}}\left(\phi_{S}+\mathrm{i} \sigma_{S}\right) .
\end{aligned}
$$

Note though that the experimental bounds on cLFV from the SUSY contributions can always be satisfied by making the SUSY states heavy enough. And at this point we have no constraint on the SUSY scale. In the future we plan to put our model into the SARAH 
package $[52,53]$ such that we can include additional constraints and give more quantitative statements. Note that if there are sources of $\mathrm{CP}$ violation $\mathrm{CP}$-even and $\mathrm{CP}$-odd scalars can mix with each other which we neglect here.

In fact, we used the SARAH code to derive the following expressions for the scalar mass matrices. In the basis $\left(\phi_{L}, \phi_{N}, \phi_{S}\right)$ the mass matrix for the CP-even sneutrinos reads

$$
m_{\tilde{\nu}^{R}}^{2}=\left(\begin{array}{ccc}
m_{\phi_{L} \phi_{L}} & m_{\phi_{R} \phi_{L}}^{T} & v_{u} \Re\left(Y_{\nu}^{T} \mu_{\mathrm{NS}}^{*}\right) \\
m_{\phi_{L} \phi_{R}} & m_{\phi_{R} \phi_{R}} & v_{X} \Re\left(\mu_{\mathrm{NS}} \lambda^{*}\right)+\Re\left(b_{\mathrm{NS}}\right) \\
v_{u} \Re\left(\mu_{\mathrm{NS}}^{T} Y_{\nu}^{*}\right) & v_{X} \Re\left(\lambda \mu_{\mathrm{NS}}^{\dagger}\right)+\Re\left(b_{\mathrm{NS}}^{T}\right) & m_{\phi_{S} \phi_{S}}
\end{array}\right),
$$

where

$$
\begin{aligned}
& m_{\phi_{L} \phi_{L}}=v_{u}^{2} \Re\left(Y_{\nu}^{T} Y_{\nu}^{*}\right)+2 \Re\left(M_{\tilde{L}}^{2}\right)+\frac{1}{2} M_{Z}^{2} \cos (2 \beta), \\
& m_{\phi_{L} \phi_{R}}=-v_{d} \Re\left(Y_{\nu} \mu_{H}^{*}\right)+v_{u} \Re\left(A_{\nu}\right), \\
& m_{\phi_{R} \phi_{R}}=\Re\left(M_{\tilde{N}^{c}}^{2}\right)+\Re\left(\mu_{\mathrm{NS}} \mu_{\mathrm{NS}}^{\dagger}\right)+v_{u}^{2} \Re\left(Y_{\nu} Y_{\nu}^{\dagger}\right), \\
& m_{\phi_{S} \phi_{S}}=\Re\left(M_{\tilde{S}}^{2}\right)+\Re\left(\mu_{\mathrm{NS}}^{T} \mu_{\mathrm{NS}}^{*}\right)+v_{X}\left(\Re\left(A_{\lambda}\right)+v_{X}\left(\Re\left(\lambda \kappa^{*}\right)+\Re\left(\lambda \lambda^{*}\right)\right)\right) .
\end{aligned}
$$

This matrix is diagonalised by $Z^{R}$ :

$$
Z^{R} m_{\tilde{\nu}^{R}}^{2} Z^{R, \dagger}=d_{\tilde{\nu}^{R}}^{2}
$$

with

$$
\left(\begin{array}{c}
\phi_{L} \\
\phi_{N} \\
\phi_{S}
\end{array}\right)=Z^{R, \dagger} \tilde{\nu}^{R} .
$$

In the basis $\left(\sigma_{L}, \sigma_{N}, \sigma_{S}\right)$ the mass matrix for the CP-odd sneutrinos reads

$$
m_{\nu^{I}}^{2}=\left(\begin{array}{ccc}
m_{\sigma_{L} \sigma_{L}} & m_{\sigma_{R} \sigma_{L}}^{T} & v_{u} \Re\left(Y_{\nu}^{T} \mu_{\mathrm{NS}}^{*}\right) \\
m_{\sigma_{L} \sigma_{R}} & m_{\sigma_{R} \sigma_{R}} & -v_{X} \Re\left(\mu_{\mathrm{NS}} \lambda^{*}\right)+\Re\left(b_{\mathrm{NS}}\right) \\
v_{u} \Re\left(\mu_{\mathrm{NS}}^{T} Y_{\nu}^{*}\right)-v_{X} \Re\left(\lambda \mu_{\mathrm{NS}}^{\dagger}\right)+\Re\left(b_{\mathrm{NS}}^{T}\right) & m_{\sigma_{S} \sigma_{S}}
\end{array}\right),
$$

where

$$
\begin{aligned}
& m_{\sigma_{L} \sigma_{L}}=v_{u}^{2} \Re\left(Y_{\nu}^{T} Y_{\nu}^{*}\right)+\Re\left(M_{\tilde{L}}^{2}\right)+\frac{1}{2} M_{Z}^{2} \cos (2 \beta), \\
& m_{\sigma_{L} \sigma_{R}}=-v_{d} \Re\left(Y_{\nu} \mu_{H}^{*}\right)+v_{u} \Re\left(A_{\nu}\right), \\
& m_{\sigma_{R} \sigma_{R}}=\Re\left(M_{\tilde{N}^{c}}^{2}\right)+\Re\left(\mu_{\mathrm{NS}} \mu_{\mathrm{NS}}^{\dagger}\right)+v_{u}^{2} \Re\left(Y_{\nu} Y_{\nu}^{\dagger}\right), \\
& m_{\sigma_{S} \sigma_{S}}=\Re\left(M_{\tilde{S}}^{2}\right)+\Re\left(\mu_{\mathrm{NS}}^{T} \mu_{\mathrm{NS}}^{*}\right)-v_{X}\left(\Re\left(A_{\lambda}\right)+v_{X}\left(\Re\left(\lambda \kappa^{*}\right)-\Re\left(\lambda \lambda^{*}\right)\right)\right) .
\end{aligned}
$$

This matrix is diagonalised by $Z^{I}$ :

$$
Z^{I} m_{\tilde{\nu}^{I}}^{2} Z^{I, \dagger}=d_{\tilde{\nu}^{I}}^{2}
$$


with

$$
\left(\begin{array}{c}
\sigma_{L} \\
\sigma_{N} \\
\sigma_{S}
\end{array}\right)=Z^{I, \dagger} \tilde{\nu}^{I}
$$

Note that the difference between the two mass matrices is proportional to the vevs $v_{N}$ and $v_{S}$ as expected.

To make the computation easier we follow a similar approach as described in [54]. Instead of treating CP-even and CP-odd scalars separately we define a larger set of sneutrino states

$$
\tilde{\nu}=\left(\begin{array}{c}
\tilde{\nu}^{R} \\
\tilde{\nu}^{I}
\end{array}\right)
$$

which has a block diagonal mass matrix

$$
m_{\tilde{\nu}}^{2}=\left(\begin{array}{cc}
m_{\tilde{\nu}^{R}}^{2} & 0 \\
0 & m_{\tilde{\nu}^{I}}^{2}
\end{array}\right)
$$

which is diagonalised by $Z^{\tilde{\nu}}$ :

$$
Z^{\tilde{\nu}} m_{\tilde{\nu}}^{2} Z^{\tilde{\nu}, \dagger}=d_{\tilde{\nu}}^{2}
$$

which is of course also block diagonal and unitary.

The vertices of the sneutrinos coupling to charginos and charged leptons, which are the relevant vertices here, can be easily reconciled from the MSSM vertices extended by right-handed neutrinos. Due to the normalisation of the fields, they get rescaled by a factor of $1 / \sqrt{2}$ and the couplings to the CP-odd scalars receive an additional factor of $i$ for the incoming vertex and a factor of $-i$ for the outgoing vertex, cf. figure 1 . Therefore, the contributions from the CP-even and CP-odd scalars can be added up. In the limit of vanishing $v_{X}$ we obtain the correct result as if there were only seven complex sneutrinos.

At this point we will not go into any more details for the full computation. There are excellent and detailed calculations for cLFV in supersymmetric inverse seesaw models in the literature, e.g. [55]. Instead, we want to discuss a bit more the qualitative features of the sneutrino mass matrices.

As we have discussed before in section 2.2 the vev $v_{X}$ is expected to be of the same order as the soft SUSY breaking parameters. Furthermore, it is a generic assumption that the soft trilinear couplings are proportional to the corresponding Yukawa couplings and hence $A_{\nu}$ is suppressed in our model. To zeroth order in $\epsilon$ that implies first of all that CP even and CP odd sneutrinos have the same mass and

$$
m_{\tilde{\nu}^{R}}^{2} \approx m_{\tilde{\nu}^{I}}^{2} \approx\left(\begin{array}{ccc}
\Re\left(M_{\tilde{L}}^{2}\right)+\frac{1}{2} M_{Z}^{2} \cos (2 \beta) & 0 & 0 \\
0 & \Re\left(M_{\tilde{N}^{c}}^{2}+\mu_{\mathrm{NS}} \mu_{\mathrm{NS}}^{\dagger}\right) & \Re\left(b_{\mathrm{NS}}\right) \\
0 & \Re\left(b_{\mathrm{NS}}^{T}\right) & \Re\left(M_{\tilde{S}}^{2}+\mu_{\mathrm{NS}}^{\dagger} \mu_{\mathrm{NS}}\right)
\end{array}\right)
$$

At this point we do not know how $b_{\mathrm{NS}}$ relates to $M_{\tilde{N}^{c}}^{2}+\mu_{\mathrm{NS}} \mu_{\mathrm{NS}}^{\dagger}$ and $M_{\tilde{S}^{c}}^{2}+\mu_{\mathrm{NS}}^{\dagger} \mu_{\mathrm{NS}}$ so that we do not know if the mixing in this sector is large or small. Since we are working 
in a basis where $\mu_{\mathrm{NS}}$ is diagonal it is also reasonable to assume that $b_{\mathrm{NS}}$ is diagonal. And hence the leading order contributions to cLFV are expected to be induced by $M_{\tilde{L}^{2}}^{2}$ and $M_{\tilde{N}^{c}}^{2}$ if the mixing between right-handed sneutrinos and scalar singlets is small. If this mixing is large $M_{\tilde{S}}^{2}$ could give sizeable cLFV in addition. It is also interesting to note that the mixing between left-handed sneutrinos and the new singlets is expected to be rather small due to a suppression by smallish Yukawa couplings.

This concludes our discussion for charged-lepton flavor violation in our model. We have seen that the non-SUSY contributions are much smaller than current bounds and the SUSY contributions can in principle be suppressed by pushing SUSY partners to the heavy limit. With the constraints given in this work so far, the SUSY partners do not necessarily have to be light. Nevertheless, this could change once we discuss potential dark matter candidates, for instance, and some interesting non-trivial interplay might emerge.

\section{Summary and conclusions}

In this work, we have proposed a minimal supersymmetric inverse seesaw model with only two generations of right-handed neutrinos $\hat{N}^{c}$, two generations of singlet fields $\hat{S}$, one symmetry breaking singlet field $\hat{X}$ and a $Z_{6}$ symmetry compared to the MSSM. With the $Z_{6}$ charge assignments listed in table 1 we have successfully forbidden some unwanted terms (e.g. $\hat{L} \hat{H}_{u} \hat{S}, \hat{H}_{u} \hat{H}_{d} \hat{N}^{c}$ in the superpotential) and retained those (e.g. $\hat{L} \hat{H}_{u} \hat{N}^{c}$ ) relevant for generating the neutrino mass. In our model we also have an intimate relation between the scale of electroweak symmetry breaking (or SUSY breaking) and the mass scales in the neutrino sector avoiding a common ad-hoc assumption in many models. This makes our model very well motivated and attractive from a model building point of view.

We have studied three different types of our model according to the mass hierarchy among $M_{S}, M_{D}$ and $\mu_{\mathrm{NS}}$. In all three types, we find three light active neutrino states with one neutrino being massless due to our minimality assumption. The mixing angles are consistent with current oscillation data which can be easily understood from the reformulation of our leading order light neutrino mass matrix in terms of the Casas-Ibarra parametrization as we have discussed. So we fulfill the minimal requirement of any neutrino mass model.

Due to the fact that the neutrino mixing matrix is now enlarged the $3 \times 3$ matrix tested in oscillations is expected to be non-unitary but our estimates for this effect is far below current bounds. Furthermore, since in our model the light active neutrinos are Majorana particles we predict neutrinoless double beta decay with an effective mass $\mathcal{O}(1) \mathrm{meV}$ and $\mathcal{O}(10) \mathrm{meV}$ for the normal and inverted hierarchy neutrino masses, respectively. These tiny numbers are experimentally challenging but on the other hand a confirmed positive signal for non-unitarity in the mixing matrix or neutrinoless double beta decay in the near future would immediately challenge our model in its minimal version.

Furthermore, we have shown qualitatively that charged lepton flavor violation with both SUSY and non-SUSY contributions can easily be below the current experimental bounds. The non-SUSY contributions are in fact far below current and future bounds and the SUSY contributions are under control since up to this point the SUSY breaking parameters can easily be in the few to several TeV region suppressing cLFV sufficiently. 
This might nevertheless change if we include further constraints. Our model has a rich dark matter and collider phenomenology which is beyond the scope of the current work but will be discussed in future publications in detail. Still we would like to use this opportunity to make a few general comments.

1. Although we did not impose $R$-parity in our model, the conventional $R$-parity violating operators, such as $L Q D^{c}, L L E^{c}, U^{c} D^{c} D^{c}$, and $L H_{u}$ are not allowed by the $Z_{6}$ symmetry.

2. We have shown that the sneutrinos from the superfields $\hat{L}, \hat{N}^{c}$, and $\hat{S}$ can all be mixed. In such a setup the lightest sneutrino could be a dark matter candidate. In the conventional MSSM, if the LSP is a left-handed sneutrino, it has been ruled out already by current direct detection experiments because of its large elastic crosssection with nuclei via $Z$-boson exchange. However, in our current model the lefthanded sneutrinos can mix with the right-handed sneutrinos and extra singlets. In such a case, the elastic scattering cross section can be suppressed or diluted to satisfy direct detection constraints.

3. The additional fermionic states which we have introduced are all expected to have masses around a $\mathrm{TeV}$. This is around the corner from the collider physics point of view and the model can be tested in current and upcoming experiments. This is indeed the main motivation for many low scale seesaw models while here it is just another appealing feature.

4. The presence of a number of sneutrinos coming from the mixing of $\tilde{\nu}_{L}, \tilde{N}^{c}$, and $\tilde{S}$ would distinguish the current model from the conventional MSSM. The sneutrinos can be directly produced via $Z$-boson exchange, or indirectly in some subsequent decays of heavier SUSY particles. If the mixing angle among the inert sneutrinos and the left-handed sneutrino is sufficiently small, the decay of the heavier sneutrinos may be prolonged such that it travels a distance without any tracks but suddenly decays with a vertex at some distance from the primary interaction point. Such an event may be detectable using the MATHUSLA detector $[56,57]$.

5. Any attempt towards a complete model of particle physics should also provide a dynamical mechanism for baryogenesis. The seesaw mechanism offers with Leptogenesis [58] an extremely popular solution for this. If this baryogenesis mechanism or another mechanism works in our model is left for another future study.

In summary our model provides a novel and rather minimal approach to supersymmetric inverse seesaw models which comes in three variants with distinct phenomenologies already in the lepton sector alone. Similar to any low scale seesaw model and in particular supersymmetric models our model provides an incredibly rich phenomenology from which we have just touched the tip of the iceberg. In fact, it can be tested at the energy, the intensity and the precision frontier as we have started to discuss here but will be discussed in greater detail in future work. 


\section{Acknowledgments}

This research was supported in parts by the Ministry of Science and Technology (MoST) of Taiwan under Grant No. MOST-105-2112-M-007-028-MY3. J.C. was supported by the National Research Foundation of Korea (NRF) grant No. NRF-2016R1E1A1A01943297.

\section{A Explicit expressions for mixing of light and heavy neutrinos}

Since the explicit expressions for the mixing between the light and the heavy neutrinos, $W$, are rather long and not insightful we present them here in the appendix. Our expressions are unitary up to order $\epsilon^{2}$ which is sufficient for our purposes. We also use $\eta$ to label the order in $\epsilon$ explicitly throughout the appendix.

To make the expressions shorter we define the abbreviations

$$
A=M_{D} \mu_{\mathrm{NS}}^{-1}, \quad B=M_{S} \mu_{\mathrm{NS}}^{-1}, \quad D=M_{D}^{T} M_{D}^{*}, \quad E=\left(\mu_{\mathrm{NS}}^{*}\right)^{-1} \mu_{\mathrm{NS}}^{-1} .
$$

For the ISS type I the mixing matrix elements are

$$
\begin{aligned}
&\left(W_{\mathrm{I}}\right)_{11}=1-1 / 2 \eta_{\mathrm{I}}^{2} A A^{\dagger}+\eta_{\mathrm{I}}^{4}\left(A A^{\dagger}\right)^{2}-1 / 4 \eta_{\mathrm{I}}^{6}\left(A A^{\dagger}\right)^{3}+1 / 2 \eta_{\mathrm{I}}^{8} A B B^{\dagger} A^{\dagger} A A^{\dagger} \\
&+\eta_{\mathrm{I}}^{8} A B E D B^{\dagger} A^{\dagger}+1 / 4 \eta_{\mathrm{I}}^{8}\left(A A^{\dagger}\right)^{4}-1 / 4 \eta_{\mathrm{I}}^{10} A A^{\dagger} A B B^{\dagger} A^{\dagger} A A^{\dagger} \\
&-1 / 2 \eta_{\mathrm{I}}^{10} A A^{\dagger} A B E D B^{\dagger} A^{\dagger}-1 / 8 \eta_{\mathrm{I}}^{10}\left(A A^{\dagger}\right)^{5} \\
&\left(W_{\mathrm{I}}\right)_{22}=+1 / 2 \eta_{\mathrm{I}}^{8} B^{\dagger} A^{\dagger} A A^{\dagger} A B+\eta_{\mathrm{I}}^{8} B^{\dagger} A^{\dagger} A B D E \\
&\left(W_{\mathrm{I}}\right)_{33}= 1-1 / 2 \eta_{\mathrm{I}}^{2} A^{\dagger} A+\eta_{\mathrm{I}}^{4}\left(A^{\dagger} A\right)^{2}-1 / 4 \eta_{\mathrm{I}}^{6}\left(A^{\dagger} A\right)^{3}+1 / 4 \eta_{\mathrm{I}}^{8}\left(A^{\dagger} A\right)^{4} \\
&-1 / 8 \eta_{\mathrm{I}}^{10}\left(A^{\dagger} A\right)^{5} \\
&\left(W_{\mathrm{I}}\right)_{12}= \eta_{\mathrm{I}}^{3} A B-\eta_{\mathrm{I}}^{5} A A^{\dagger} A B-\eta_{\mathrm{I}}^{5} A B D E+1 / 4 \eta_{\mathrm{I}}^{7}\left(A A^{\dagger}\right)^{2} A B \\
&+1 / 2 \eta_{\mathrm{I}}^{7} A A^{\dagger} A B D E-1 / 2 \eta_{\mathrm{I}}^{9}\left(A A^{\dagger}\right)^{3} A B-\eta_{\mathrm{I}}^{9}\left(A A^{\dagger}\right)^{2} A B D E \\
&\left(W_{\mathrm{I}}\right)_{13}=-\eta_{\mathrm{I}} A+\eta_{\mathrm{I}}^{3} A A^{\dagger} A-3 / 4 \eta_{\mathrm{I}}^{5}\left(A A^{\dagger}\right)^{2} A+1 / 8 \eta_{\mathrm{I}}^{7}\left(A A^{\dagger}\right)^{3} A \\
&\left.-1 / 4 \eta_{\mathrm{I}}^{9} A A^{\dagger}\right)^{4} A \\
&\left(W_{\mathrm{I}}\right)_{23}= 1 / 4 \eta_{\mathrm{I}}^{8} B^{\dagger}\left(A^{\dagger} A\right)^{3}, \\
&\left(W_{\mathrm{I}}\right)_{21}=-\eta_{\mathrm{I}}^{3} B^{\dagger} A^{\dagger}+1 / 2 \eta_{\mathrm{I}}^{5} B^{\dagger} A^{\dagger} A A^{\dagger}+\eta_{\mathrm{I}}^{5} E D B^{\dagger} A^{\dagger} \\
&\left(W_{\mathrm{I}}\right)_{31}= \eta_{\mathrm{I}} A^{\dagger}-\eta_{\mathrm{I}}^{3} A^{\dagger} A A^{\dagger}+3 / 4 \eta_{\mathrm{I}}^{5}\left(A^{\dagger} A\right)^{2} A^{\dagger}-1 / 8 \eta_{\mathrm{I}}^{7}\left(A^{\dagger} A\right)^{3} A^{\dagger} \\
&+1 / 2 \eta_{\mathrm{I}}^{9} A^{\dagger} A B B^{\dagger} A^{\dagger} A A^{\dagger}+\eta_{\mathrm{I}}^{9} A^{\dagger} A B E D B^{\dagger} A^{\dagger}+1 / 4 \eta_{\mathrm{I}}^{9}\left(A^{\dagger} A\right)^{4} A^{\dagger}, \\
&\left(W_{\mathrm{I}}\right)_{32}= \eta_{\mathrm{I}}^{4} A^{\dagger} A B-1 / 2 \eta_{\mathrm{I}}^{6}\left(A^{\dagger} A\right)^{2} B-\eta_{\mathrm{I}}^{6} A^{\dagger} A B D E+1 / 2 \eta_{\mathrm{I}}^{8}\left(A^{\dagger} A\right)^{3} B \\
&+\eta_{\mathrm{I}}^{8}\left(A^{\dagger} A\right)^{2} B D E-1 / 4 \eta_{\mathrm{I}}^{10}\left(A^{\dagger} A\right)^{4} B-1 / 2 \eta_{\mathrm{I}}^{10}\left(A^{\dagger} A\right)^{3} B D E
\end{aligned}
$$

where we have quoted for convenience the orders in $\eta_{\mathrm{I}}$ explicitly. 
For the ISS type II the mixing matrix elements are

$$
\begin{aligned}
\left(W_{\mathrm{II}}\right)_{11}= & 1-1 / 2 \eta_{\mathrm{II}}^{2} A A^{\dagger}+\eta_{\mathrm{II}}^{4}\left(A A^{\dagger}\right)^{2}+1 / 2 \eta_{\mathrm{II}}^{6} A B B^{\dagger} A^{\dagger} A A^{\dagger}+\eta_{\mathrm{II}}^{6} A B E D B^{\dagger} A^{\dagger} \\
& -1 / 4 \eta_{\mathrm{II}}^{8} A A^{\dagger} A B B^{\dagger} A^{\dagger} A A^{\dagger}-1 / 2 \eta_{\mathrm{II}}^{8} A A^{\dagger} A B E D B^{\dagger} A^{\dagger} \\
\left(W_{\mathrm{II}}\right)_{22}= & 1+1 / 2 \eta_{\mathrm{II}}^{6} B^{\dagger} A^{\dagger} A A^{\dagger} A B+\eta_{\mathrm{II}}^{6} B^{\dagger} A^{\dagger} A B D E \\
\left(W_{\mathrm{II}}\right)_{33}= & 1-1 / 2 \eta_{\mathrm{II}}^{2} A^{\dagger} A+\eta_{\mathrm{II}}^{4} A^{\dagger} A A^{\dagger} A \\
\left(W_{\mathrm{II}}\right)_{12}= & \eta_{\mathrm{II}}^{2} A B-\eta_{\mathrm{II}}^{4} A A^{\dagger} A B-\eta_{\mathrm{II}}^{4} A B D E+1 / 4 \eta_{\mathrm{II}}^{6}\left(A A^{\dagger}\right)^{2} A B \\
& +1 / 2 \eta_{\mathrm{II}}^{6} A A^{\dagger} A B D E-1 / 2 \eta_{\mathrm{II}}^{8}\left(A A^{\dagger}\right)^{3} A B-\eta_{\mathrm{II}}^{8}\left(A A^{\dagger}\right)^{2} A B D E \\
\left(W_{\mathrm{II}}\right)_{13}= & -\eta_{\mathrm{II}} A+\eta_{\mathrm{II}}^{3} A A^{\dagger} A-1 / 2 \eta_{\mathrm{II}}^{5}\left(A A^{\dagger}\right)^{2} A \\
\left(W_{\mathrm{II}}\right)_{23}= & -\eta_{\mathrm{II}}^{5} B^{\dagger}\left(A^{\dagger} A\right)^{2} \\
\left(W_{\mathrm{II}}\right)_{21}= & -\eta_{\mathrm{II}}^{2} B^{\dagger} A^{\dagger}+1 / 2 \eta_{\mathrm{II}}^{4} B^{\dagger} A^{\dagger} A A^{\dagger}+\eta_{\mathrm{II}}^{4} E D B^{\dagger} A^{\dagger} \\
\left(W_{\mathrm{II}}\right)_{31}= & \eta_{\mathrm{II}} A^{\dagger}-\eta_{\mathrm{II}}^{3} A^{\dagger} A A^{\dagger}+1 / 2 \eta_{\mathrm{II}}^{5}\left(A^{\dagger} A\right)^{2} A^{\dagger}+1 / 2 \eta_{\mathrm{II}}^{7} A^{\dagger} A B B^{\dagger} A^{\dagger} A A^{\dagger} \\
& +\eta_{\mathrm{II}}^{7} A^{\dagger} A B E D B^{\dagger} A^{\dagger} \\
\left(W_{\mathrm{II}}\right)_{32}= & \eta_{\mathrm{II}}^{3} A^{\dagger} A B-1 / 2 \eta_{\mathrm{II}}^{5}\left(A^{\dagger} A\right)^{2} B-\eta_{\mathrm{II}}^{5} A^{\dagger} A B D E+1 / 2 \eta_{\mathrm{II}}^{7}\left(A^{\dagger} A\right)^{3} B \\
& +\eta_{\mathrm{II}}^{7}\left(A^{\dagger} A\right)^{2} B D E-1 / 4 \eta_{\mathrm{II}}^{9}\left(A^{\dagger} A\right)^{4} B-1 / 2 \eta_{\mathrm{II}}^{9}\left(A^{\dagger} A\right)^{3} B D E
\end{aligned}
$$

where we have quoted for convenience the orders in $\eta_{\text {II }}$ explicitly.

For the ISS type III the mixing matrix elements are

$$
\begin{aligned}
& \left(W_{\mathrm{III}}\right)_{11}=1, \\
& \left(W_{\mathrm{III}}\right)_{22}=1, \\
& \left(W_{\mathrm{III}}\right)_{33}=1, \\
& \left(W_{\mathrm{III}}\right)_{12}=\eta_{\mathrm{III}}^{3} A B, \\
& \left(W_{\mathrm{III}}\right)_{13}=-\eta_{\mathrm{III}}^{2} A, \\
& \left(W_{\mathrm{III}}\right)_{23}=\mathcal{O}\left(\eta_{\mathrm{III}}^{13}\right), \\
& \left(W_{\mathrm{III}}\right)_{21}=-\eta_{\mathrm{III}}^{3} B^{\dagger} A^{\dagger}, \\
& \left(W_{\mathrm{III}}\right)_{31}=-\eta_{\mathrm{III}}^{2} A^{\dagger}, \\
& \left(W_{\mathrm{III}}\right)_{32}=\eta_{\mathrm{III}}^{5} A^{\dagger} A B,
\end{aligned}
$$

where we have quoted for convenience the orders in $\eta_{\text {III }}$ explicitly.

Open Access. This article is distributed under the terms of the Creative Commons Attribution License (CC-BY 4.0), which permits any use, distribution and reproduction in any medium, provided the original author(s) and source are credited.

\section{References}

[1] H.E. Haber and G.L. Kane, The search for supersymmetry: probing physics beyond the standard model, Phys. Rept. 117 (1985) 75 [INSPIRE].

[2] D.V. Forero, M. Tortola and J.W.F. Valle, Neutrino oscillations refitted, Phys. Rev. D 90 (2014) 093006 [arXiv:1405.7540] [INSPIRE]. 
[3] M. Hirsch, M.A. Diaz, W. Porod, J.C. Romao and J.W.F. Valle, Neutrino masses and mixings from supersymmetry with bilinear $R$ parity violation: a theory for solar and atmospheric neutrino oscillations, Phys. Rev. D 62 (2000) 113008 [Erratum ibid. D 65 (2002) 119901] [hep-ph/0004115] [INSPIRE].

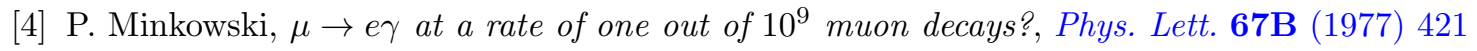
[INSPIRE].

[5] T. Yanagida, Horizontal symmetry and masses of neutrinos, in the proceedings of the Workshop on unified theory and baryon number in the universe, O. Sawada and A. Sugamoto eds., KEK, Tsukuba, Japan (1979).

[6] T. Yanagida, Horizontal symmetry and masses of neutrinos, Prog. Theor. Phys. 64 (1980) 1103 [INSPIRE].

[7] M. Gell-Mann, P. Ramond and R. Slansky, Complex spinors and unified theories, Conf. Proc. C 790927 (1979) 315 [arXiv: 1306.4669] [INSPIRE].

[8] P. Ramond, The family group in grand unified theories, talk given at Sanibel Symposium, February 25-March 2, Palm Coast U.S.A. (1979), hep-ph/9809459 [INSPIRE].

[9] S.L. Glashow, The future of elementary particle physics, in Quarks and leptons, Cargèse lectures, M. Lévy et al. eds., Plenum Press, New York, U.S.A. (1980).

[10] R.N. Mohapatra and G. Senjanović, Neutrino mass and spontaneous parity violation, Phys. Rev. Lett. 44 (1980) 912 [INSPIRE].

[11] J. Schechter and J.W.F. Valle, Neutrino decay and spontaneous violation of lepton number, Phys. Rev. D 25 (1982) 774 [INSPIRE].

[12] R.N. Mohapatra, Mechanism for understanding small neutrino mass in superstring theories, Phys. Rev. Lett. 56 (1986) 561 [INSPIRE].

[13] R.N. Mohapatra and J.W.F. Valle, Neutrino mass and baryon number nonconservation in superstring models, Phys. Rev. D 34 (1986) 1642 [InSPIRE].

[14] A. Das, P.S. Bhupal Dev and N. Okada, Direct bounds on electroweak scale pseudo-Dirac neutrinos from $\sqrt{s}=8 \mathrm{TeV}$ LHC data, Phys. Lett. B 735 (2014) 364 [arXiv:1405.0177] [INSPIRE].

[15] A. Das and N. Okada, Improved bounds on the heavy neutrino productions at the LHC, Phys. Rev. D 93 (2016) 033003 [arXiv:1510.04790] [INSPIRE].

[16] M.L. Mangano et al., Physics at a 100 TeV pp collider: standard model processes, CERN Yellow Report (2017) 1 [arXiv: 1607.01831] [INSPIRE].

[17] A. Das and N. Okada, Inverse seesaw neutrino signatures at the LHC and ILC, Phys. Rev. D 88 (2013) 113001 [arXiv: 1207.3734] [INSPIRE].

[18] S. Antusch and O. Fischer, Testing sterile neutrino extensions of the Standard Model at future lepton colliders, JHEP 05 (2015) 053 [arXiv: 1502.05915] [INSPIRE].

[19] FCC-ee study Team collaboration, A. Blondel et al., Search for heavy right handed neutrinos at the FCC-ee, Nucl. Part. Phys. Proc. 273-275 (2016) 1883 [arXiv:1411.5230] [INSPIRE].

[20] S. Antusch, E. Cazzato and O. Fischer, Sterile neutrino searches at future $e^{-} e^{+}, p p$ and $e^{-} p$ colliders, Int. J. Mod. Phys. A 32 (2017) 1750078 [arXiv: 1612.02728] [INSPIRE]. 
[21] A. Atre, T. Han, S. Pascoli and B. Zhang, The search for heavy Majorana neutrinos, JHEP 05 (2009) 030 [arXiv:0901.3589] [INSPIRE].

[22] E. Arganda, M.J. Herrero, X. Marcano and C. Weiland, Enhancement of the lepton flavor violating Higgs boson decay rates from SUSY loops in the inverse seesaw model, Phys. Rev. D 93 (2016) 055010 [arXiv: 1508.04623] [INSPIRE].

[23] F. Deppisch and J.W.F. Valle, Enhanced lepton flavor violation in the supersymmetric inverse seesaw model, Phys. Rev. D 72 (2005) 036001 [hep-ph/0406040] [InSPIRE].

[24] C. Arina, F. Bazzocchi, N. Fornengo, J.C. Romao and J.W.F. Valle, Minimal supergravity sneutrino dark matter and inverse seesaw neutrino masses, Phys. Rev. Lett. 101 (2008) 161802 [arXiv: 0806 .3225] [INSPIRE].

[25] P.S. Bhupal Dev, S. Mondal, B. Mukhopadhyaya and S. Roy, Phenomenology of Light Sneutrino Dark Matter in cMSSM/mSUGRA with Inverse Seesaw, JHEP 09 (2012) 110 [arXiv: 1207.6542] [INSPIRE].

[26] M. Hirsch, T. Kernreiter, J.C. Romao and A. Villanova del Moral, Minimal supersymmetric inverse seesaw: neutrino masses, lepton flavour violation and LHC phenomenology, JHEP 01 (2010) 103 [arXiv:0910.2435] [INSPIRE].

[27] S. Khalil, H. Okada and T. Toma, Right-handed sneutrino dark matter in supersymmetric B-L model, JHEP 07 (2011) 026 [arXiv: 1102.4249] [INSPIRE].

[28] M. Hirsch, M. Malinsky, W. Porod, L. Reichert and F. Staub, Hefty MSSM-like light Higgs in extended gauge models, JHEP 02 (2012) 084 [arXiv:1110.3037] [INSPIRE].

[29] M. Hirsch, W. Porod, L. Reichert and F. Staub, Phenomenology of the minimal supersymmetric $\mathrm{U}(1)_{B-L} \times \mathrm{U}(1)_{R}$ extension of the standard model, Phys. Rev. D 86 (2012) 093018 [arXiv: 1206.3516] [INSPIRE].

[30] V. De Romeri and M. Hirsch, Sneutrino dark matter in low-scale seesaw scenarios, JHEP 12 (2012) 106 [arXiv:1209.3891] [INSPIRE].

[31] Z. Kang, J. Li, T. Li, T. Liu and J.M. Yang, The maximal $\mathrm{U}(1)_{L}$ inverse seesaw from $d=5$ operator and oscillating asymmetric Sneutrino dark matter, Eur. Phys. J. C 76 (2016) 270 [arXiv: 1102.5644] [INSPIRE].

[32] S.-L. Chen and Z. Kang, Oscillating asymmetric sneutrino dark matter from the maximally $\mathrm{U}(1)_{L}$ supersymmetric inverse seesaw, Phys. Lett. B 761 (2016) 296 [arXiv:1512.08780] [INSPIRE].

[33] F. Bazzocchi, D.G. Cerdeno, C. Muñoz and J.W.F. Valle, Calculable inverse-seesaw neutrino masses in supersymmetry, Phys. Rev. D 81 (2010) 051701 [arXiv:0907.1262] [INSPIRE].

[34] P.S.B. Dev and R.N. Mohapatra, TeV scale inverse seesaw in $\mathrm{SO}(10)$ and leptonic non-unitarity effects, Phys. Rev. D 81 (2010) 013001 [arXiv:0910.3924] [INSPIRE].

[35] H. An, P.S.B. Dev, Y. Cai and R.N. Mohapatra, Sneutrino dark matter in gauged inverse seesaw models for neutrinos, Phys. Rev. Lett. 108 (2012) 081806 [arXiv:1110.1366] [INSPIRE].

[36] A. Abada and M. Lucente, Looking for the minimal inverse seesaw realisation, Nucl. Phys. B 885 (2014) 651 [arXiv:1401.1507] [INSPIRE].

[37] S.P. Martin, A Supersymmetry primer, Adv. Ser. Direct. High Energy Phys. 21 (2010) 1 [Adv. Ser. Direct. High Energy Phys. 18 (1998) 1] [hep-ph/9709356] [INSPIRE]. 
[38] G. 't Hooft, Naturalness, chiral symmetry, and spontaneous chiral symmetry breaking, NATO Sci. Ser. B 59 (1980) 135.

[39] E. Fernandez-Martinez, J. Hernandez-Garcia and J. Lopez-Pavon, Global constraints on heavy neutrino mixing, JHEP 08 (2016) 033 [arXiv: 1605.08774] [INSPIRE].

[40] J.A. Casas and A. Ibarra, Oscillating neutrinos and $\mu \rightarrow e, \gamma$, Nucl. Phys. B 618 (2001) 171 [hep-ph/0103065] [INSPIRE].

[41] H. Päs and W. Rodejohann, Neutrinoless double beta decay, New J. Phys. 17 (2015) 115010 [arXiv: 1507.00170] [INSPIRE].

[42] KamLAnD-Zen collaboration, A. Gando et al., Search for Majorana Neutrinos near the Inverted Mass Hierarchy Region with KamLAND-Zen, Phys. Rev. Lett. 117 (2016) 082503 [arXiv: 1605.02889] [INSPIRE].

[43] M.C. Gonzalez-Garcia, M. Maltoni and T. Schwetz, Updated fit to three neutrino mixing: status of leptonic CP-violation, JHEP 11 (2014) 052 [arXiv:1409.5439] [INSPIRE].

[44] R.N. Mohapatra, New contributions to neutrinoless double beta decay in supersymmetric theories, Phys. Rev. D 34 (1986) 3457 [InSPIRE].

[45] A. Faessler, M. González, S. Kovalenko and F. Šimkovic, Arbitrary mass Majorana neutrinos in neutrinoless double beta decay, Phys. Rev. D 90 (2014) 096010 [arXiv:1408.6077] [INSPIRE].

[46] N. Haba, H. Ishida and Y. Yamaguchi, Naturalness and lepton number/flavor violation in inverse seesaw models, JHEP 11 (2016) 003 [arXiv:1608.07447] [INSPIRE].

[47] T.P. Cheng and L.-F. Li, $\mu \rightarrow$ e $\gamma$ in theories with Dirac and Majorana neutrino mass terms, Phys. Rev. Lett. 45 (1980) 1908 [INSPIRE].

[48] S.T. Petcov, The processes $\mu \rightarrow e \gamma, \mu \rightarrow e e \bar{e}, \nu^{\prime} \rightarrow \nu \gamma$ in the Weinberg-Salam model with neutrino mixing, Sov. J. Nucl. Phys. 25 (1977) 340 [Erratum ibid. 25 (1977) 698] [Yad. Fiz. 25 (1977) 641] [Erratum ibid. 25 (1977) 1336] [INSPIRE].

[49] MEG collaboration, A.M. Baldini et al., Search for the lepton flavour violating decay $\mu^{+} \rightarrow \mathrm{e}^{+} \gamma$ with the full dataset of the MEG experiment, Eur. Phys. J. C 76 (2016) 434 [arXiv: 1605.05081] [INSPIRE].

[50] J. Hisano, T. Moroi, K. Tobe and M. Yamaguchi, Lepton flavor violation via right-handed neutrino Yukawa couplings in supersymmetric standard model, Phys. Rev. D 53 (1996) 2442 [hep-ph/9510309] [INSPIRE].

[51] J. Hisano, T. Moroi, K. Tobe, M. Yamaguchi and T. Yanagida, Lepton flavor violation in the supersymmetric standard model with seesaw induced neutrino masses, Phys. Lett. B 357 (1995) 579 [hep-ph/9501407] [INSPIRE].

[52] F. Staub, SARAH, arXiv:0806.0538 [INSPIRE].

[53] F. Staub, SARAH 4: a tool for (not only SUSY) model builders, Comput. Phys. Commun. 185 (2014) 1773 [arXiv: 1309.7223] [INSPIRE].

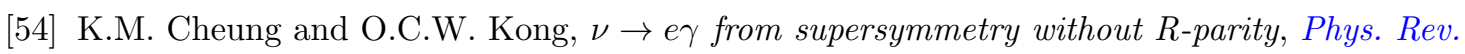
D 64 (2001) 095007 [hep-ph/0101347].

[55] A. Abada, M.E. Krauss, W. Porod, F. Staub, A. Vicente and C. Weiland, Lepton flavor violation in low-scale seesaw models: SUSY and non-SUSY contributions, JHEP 11 (2014) 048 [arXiv: 1408.0138] [INSPIRE]. 
[56] J.P. Chou, D. Curtin and H.J. Lubatti, New detectors to explore the lifetime frontier, Phys. Lett. B 767 (2017) 29 [arXiv: 1606. 06298] [INSPIRE].

[57] D. Curtin and M.E. Peskin, Analysis of long lived particle decays with the MATHUSLA detector, arXiv: 1705.06327 [INSPIRE].

[58] M. Fukugita and T. Yanagida, Baryogenesis without grand unification, Phys. Lett. B 174 (1986) 45 [INSPIRE]. 